\title{
An Ensemble-Guided Approach Identifies ClpP as a Major Regulator of Transcript Levels in Nitric Oxide-Stressed Escherichia coli
}

Jonathan L. Robinson and Mark P. Brynildsen*

Department of Chemical and Biological Engineering, Princeton University, Princeton, New Jersey, 08544, United States of America.

*Corresponding author

Phone: +1 609-258-1995

Fax: +1 609-258-0211

Email: mbrynild@princeton.edu

Postal address:

205 Hoyt Laboratory

25 William Street

Princeton, NJ 08544

U.S.A. 


\begin{abstract}
The importance of $\mathrm{NO} \bullet$ to immunity is highlighted by the diversity of pathogens that require NO•-defensive systems to establish infections. Proteases have been identified to aid pathogens in surviving macrophage attack, inspiring us to investigate their role during NO• stress in Escherichia coli. We discovered that the elimination of ClpP largely impaired NO• detoxification by $E$. coli. Using a quantitative model of $\mathrm{NO} \bullet$ stress, we employed an ensembleguided approach to identify the underlying mechanism. Iterations of in silico analyses and corresponding experiments identified the defect to result from deficient transcript levels of $h m p$, which encodes $\mathrm{NO} \cdot$ dioxygenase. Interestingly, the defect was not confined to $h m p$, as $\Delta c l p P$ imparted widespread perturbations to the expression of NO•-responsive genes. This work identified a target for anti-infective therapies based on disabling NO• defenses, and demonstrated the utility of model-based approaches for exploring the complex, systems-level stress exerted by $\mathrm{NO} \cdot$
\end{abstract}

Keywords: nitric oxide; Escherichia coli; ClpP protease; kinetic modeling; nitrosative stress; Hmp 


\section{Introduction}

$\mathrm{NO} \bullet$ is an important signaling molecule in humans, modulating physiological functions ranging from vasodilation and neurotransmission to innate immunity [1-3]. As part of the immune response, $\mathrm{NO} \bullet$ is synthesized by phagocytic cells, such as macrophages, to disable pathogens [3,4] through inhibition of processes such as respiration [5], amino acid biosynthesis [6], and the tricarboxylic acid (TCA) cycle [7]. The importance of NO• in the immune response is emphasized by the growing list of pathogens, including Pseudomonas aeruginosa [8], Vibrio cholerae [9], Mycobacterium tuberculosis [10], Salmonella enterica serovar Typhimurium [11], and pathogenic strains of $E$. coli $[12,13]$, for which $\mathrm{NO} \bullet$ detoxification or repair systems have been identified as virulence factors [14]. In uropathogenic E. coli (UPEC), for example, a mutant lacking NO• dioxygenase $(\Delta h m p)$ was outcompeted by wild-type (WT) in a mouse infection model [12]. More recently, the presence of a functional NO• reductase (NorV) in enterohemorrhagic E. coli (EHEC) strains has been found to correlate with higher incidences of hemolytic uremic syndrome (HUS), a life-threatening condition [15]. Further study found that EHEC possessing functional nor $V$ exhibited increased survival within macrophages compared to strains harboring the inactive norVs gene [13]. Furthermore, murine infection studies have shown that deletion or inhibition of inducible nitric oxide synthase (iNOS), the enzyme responsible for NO• production in phagocytes [16], increases the bacterial load and mortality rate of infected hosts for a wide variety of pathogens [7,17-19]. These data suggest that sabotaging bacterial NO• defenses could constitute an effective anti-infective strategy for many pathogens. However, known inhibitors for the obvious network targets, such as NO• dioxygenase (Hmp), are not therapeutically applicable, as they face issues such as toxicity toward humans (e.g., cyanide [20]) or poor transport into Gram-negative bacteria (e.g., imidazole [21]). Therefore, additional targets 
that could be leveraged to disable bacterial $\mathrm{NO} \bullet$ defenses are necessary to realize therapies based on potentiation of $\mathrm{NO} \cdot$ toxicity in pathogens.

Bacterial proteases have recently been identified as virulence factors for numerous pathogens [22], and more specifically, been found to enhance survival within macrophages [23,24]. For example, the Hip1 protease in M. tuberculosis was identified to aid in the evasion of immune attack by suppressing the proinflammatory response and the activation of macrophages $[25,26]$. The ClpP protease has been found to be essential for the survival of both Listeria monocytogenes [27] and Streptococcus pneumonia [24] in macrophages, whereas the Lon protease was found to be important for survival of $S$. Typhimurium in macrophages [23]. These studies, in conjunction with the knowledge that $\mathrm{NO} \bullet$ is a major component of the macrophage antimicrobial arsenal, suggested that proteases may be important mediators of bacterial NO• defense systems. However, it was unknown whether proteases participate in the NO• stress response of E. coli. Considering the importance of NO• tolerance to EHEC and UPEC virulence $[12,13]$, we investigated whether proteases were involved in the NO• defense network of E. coli. Here, we evaluated the NO• sensitivity of E. coli deficient in each of the three major ATP-dependent proteases (Clp, Lon, and Hsl), and discovered that $\Delta c l p P$ exhibited a substantial increase in $\mathrm{NO} \bullet-$ mediated stasis, and decrease in $\mathrm{NO} \bullet$ clearance rate, compared to WT. We found the phenotype to also be present in EHEC, and mechanistically explored the result using a quantitative modeling approach to guide experimentation. Specifically, a kinetic model of the $E$. coli $\mathrm{NO} \bullet$ biochemical network was used to generate an ensemble of mechanistic hypotheses that could explain the $\triangle c l p P$ phenotype, and was subjected to experimentally-tractable perturbations in silico to identify conditions that would allow for discrimination between mechanisms. The in silico perturbations were executed experimentally and the results were used to eliminate 
mechanisms that were inconsistent with the data. This process was iterated until we determined that a deficiency in $h m p$ transcript levels was responsible for the increased NO• sensitivity of $\Delta c l p P$. Using RNA sequencing (RNA-Seq), we found that the deletion of ClpP had a widespread impact on transcript levels under $\mathrm{NO} \bullet$ stress. These results demonstrate the utility of ensembleguided approaches for the mechanistic dissection of phenotypes associated with the broadly reactive immune antimicrobial $\mathrm{NO} \bullet$, and suggest that $\mathrm{ClpP}$ may be an attractive target for therapies to treat pathogenic E. coli, such as EHEC.

\section{Results}

\subsection{NO• sensitivity of $E$. coli protease mutants}

To determine if protease activity contributes to $E$. coli NO• defense, single gene knockout mutants of each of the three major ATP-dependent proteases $(\Delta c l p P, \Delta l o n$, and $\Delta h s l V)$ were evaluated for $\mathrm{NO} \bullet$ sensitivity relative to the WT (Fig. 1A). A fourth protease, FtsH, was excluded from the investigation because it is required for growth [28]. Cultures were grown to exponential phase, and treated with 2 mM DPTA NONOate, which dissociates to release 2 NO• molecules per parent compound. In the absence of $\mathrm{NO} \bullet$ stress, growth was nearly identical among the protease mutants and WT. Treatment with DPTA NONOate resulted in a similar 3 to $4 \mathrm{~h}$ of growth arrest for WT, $\Delta l o n$, and $\Delta h s l V$, but the recovery of $\Delta c l p P$ was largely impaired, requiring twice as long $(\sim 8 \mathrm{~h})$ to resume growth. The increased $\mathrm{NO} \bullet$ sensitivity of $\Delta c l p P$ suggested that ClpP protease activity plays an important role in the E. coli response to, and/or recovery from $\mathrm{NO} \bullet$ stress. To investigate this phenomenon, NO• clearance was measured for WT and each of the three protease mutants. 

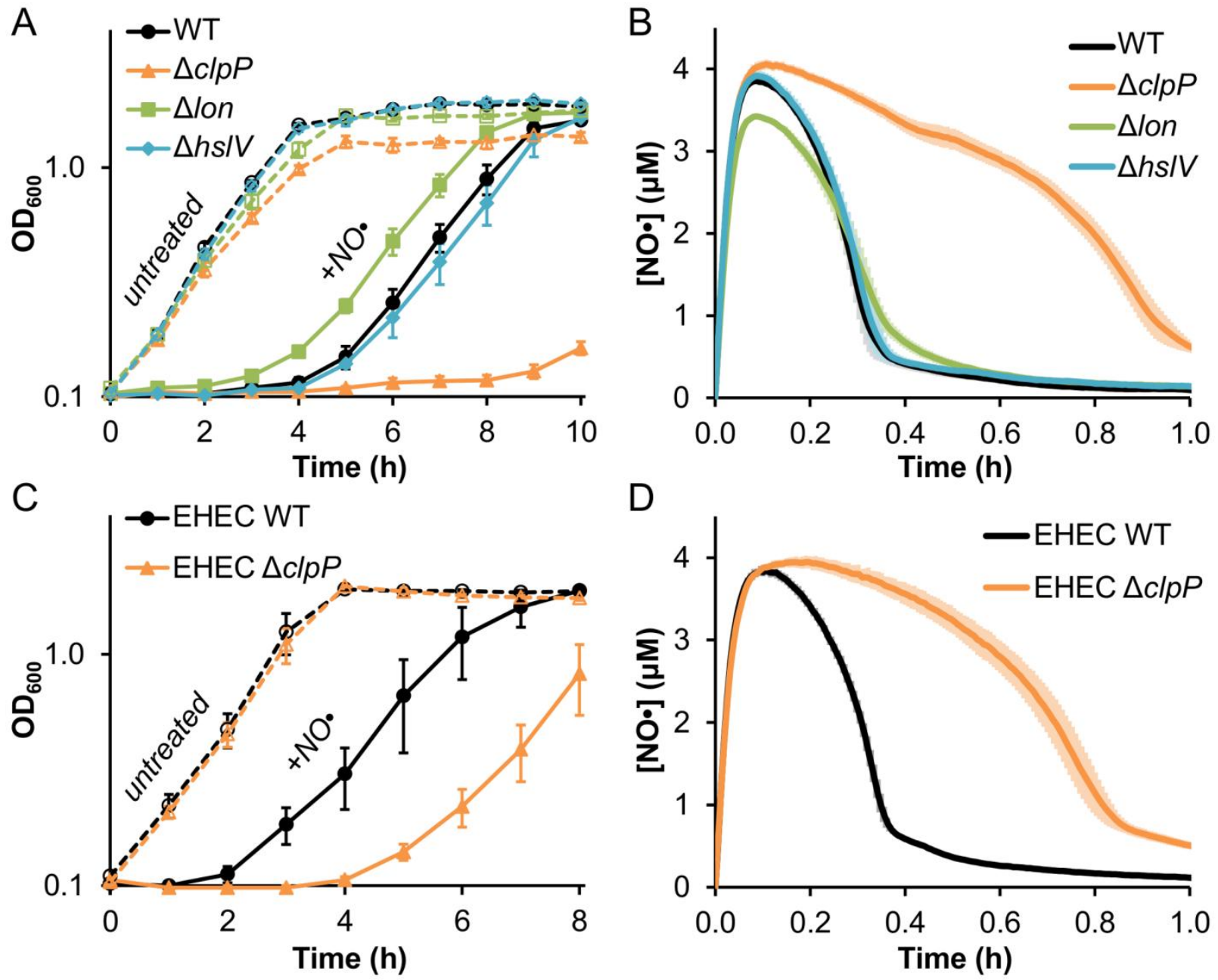

Figure 1. Deletion of $c l p P$ impairs NO• defenses in E. coli K-12 and EHEC. (A) WT E. coli $\mathrm{K}-12$ and three protease mutants $(\Delta c l p P, \Delta l o n$, and $\Delta h s l V)$ were grown aerobically in MOPS + glucose media from an initial $\mathrm{OD}_{600}$ of 0.01 to mid-exponential phase $\left(\mathrm{OD}_{600}=0.2\right)$, diluted to an $\mathrm{OD}_{600}$ of 0.1 in fresh media, and treated with $2 \mathrm{mM}$ DPTA NONOate (solid lines, filled markers) or left untreated (dashed lines, open markers), and growth was monitored hourly. (B) Cultures were grown aerobically to exponential phase, diluted to an $\mathrm{OD}_{600}$ of 0.05 in a bioreactor, immediately treated with $250 \mu \mathrm{M}$ DPTA NONOate, and [NO ${ }^{\bullet}$ was monitored continuously for $1 \mathrm{~h}$ post-dose. (C) WT EHEC TUV93-0 and its $\triangle c l p P$ mutant were grown and treated identically as in (A), except using a DPTA NONOate concentration of $1 \mathrm{mM}$. (D) WT and $\triangle c l p P$ EHEC were treated identically as described in $(\mathbf{B})$. Data points are the mean of at least 3 independent experiments, with error bars representing the standard error of the mean.

Consistent with the growth phenotype, $\Delta l o n$ and $\Delta h s l V$ exhibited $\mathrm{NO} \bullet$ detoxification activity similar to that of WT, whereas $\Delta c l p P \mathrm{NO} \bullet$ clearance was over two-fold slower (Fig. 1B). This phenotype was complemented with a plasmid-borne copy of $c l p P$ expressed from its native 
promoter (pJR01), unequivocally demonstrating that the phenotype was associated with loss of ClpP (Fig. S1).

To assess whether the phenotype is present in pathogenic E. coli, we performed a similar analysis of $\mathrm{NO} \bullet$ sensitivity for $\mathrm{EHEC}$ and its $\Delta c l p P$ mutant. $\mathrm{NO} \bullet$ has been suggested to play a critical role in EHEC pathogenesis, where mutants lacking the detoxification enzyme NO• reductase (NorV) were found to exhibit reduced survival in macrophages [13], and infection outbreaks involving strains possessing a functional NorV (as opposed to the inactive NorVs) correlated with a higher incidence of hemolytic uremic syndrome (HUS) [15]. Similar to E. coli K-12, EHEC $\Delta c l p P$ demonstrated an impaired ability to cope with $\mathrm{NO} \bullet$ stress, exhibiting over a two-fold increase in NO•-induced stasis (Fig. 1C) and NO• clearance time (Fig. 1D).

\subsection{Dissection of the $\triangle c l p P$ phenotype with an ensemble-guided approach}

\subsubsection{Model ensemble generation}

The promiscuity and reactivity of $\mathrm{NO} \bullet$ and its reaction products give rise to a broad, complex biochemical reaction network that can be difficult to interrogate without the use of mathematical models [3,14,29]. Within E. coli, NO• may nitrosylate iron-sulfur clusters, bind reversibly to cytochrome oxidase hemes, or undergo enzymatic detoxification by Hmp (aerobic), NorV (NO• reductase; anaerobic), or NrfA (nitrite reductase; anaerobic). Further, NO• can react with $\mathrm{O}_{2}$ or superoxide to form additional reactive nitrogen species (e.g., $\mathrm{NO}_{2}^{\bullet}, \mathrm{N}_{2} \mathrm{O}_{3}, \mathrm{ONOO}^{-}$), which can deaminate DNA bases, nitrosate thiols, react with protein residues such as tyrosine and tryptophan, or undergo hydrolysis to yield $\mathrm{NO}_{2}{ }^{-}$and $\mathrm{NO}_{3}{ }^{-}[3,14,29]$. Our previous work has demonstrated the utility of computational modeling for quantitatively studying the NO• biochemical network of $E$. coli and its response to various genetic and environmental 
perturbations $[29,30]$. We therefore employed the model to mechanistically dissect the impact of $\Delta c l p P$ on the $E$. coli $\mathrm{NO} \cdot$ response network.

In brief, the $E$. coli $\mathrm{NO} \bullet$ model is comprised of 189 reactions, 139 species, 183 kinetic parameters, and 2 compartments: intracellular and extracellular (growth medium). Kinetic parameter values, reaction network structure, and initial species concentrations were obtained from literature, measured experimentally, or in some cases classified as uncertain and approximated using an optimization procedure that minimized the sum squared residual error (SSR) between model simulations and experimental measurements (Text S1). A parametric analysis was subsequently conducted to identify the uncertain parameters as informed or uninformed by the optimization procedure (Text S1). All further analyses were based on parameters obtained from literature, measured experimentally, or informed during the optimization procedure.

The [NO•] curve simulated by the model was in excellent agreement with experimental measurements for the WT culture (Fig. 2A). Since ClpP is a novel component of the NO• response network, and thus absent from the knowledgebase upon which the model was constructed [29], the model incorrectly predicted WT [NO•] dynamics for $\Delta c l p P$. To identify the mechanism by which $\Delta c l p P$ impairs $\mathrm{NO} \bullet$ detoxification, parameter constraints were released one by one, and allowed to vary (increase or decrease) within two-fold of their original value to determine those with the greatest capacity to reconcile simulations with experimental data from $\Delta c l p P$ cultures. 

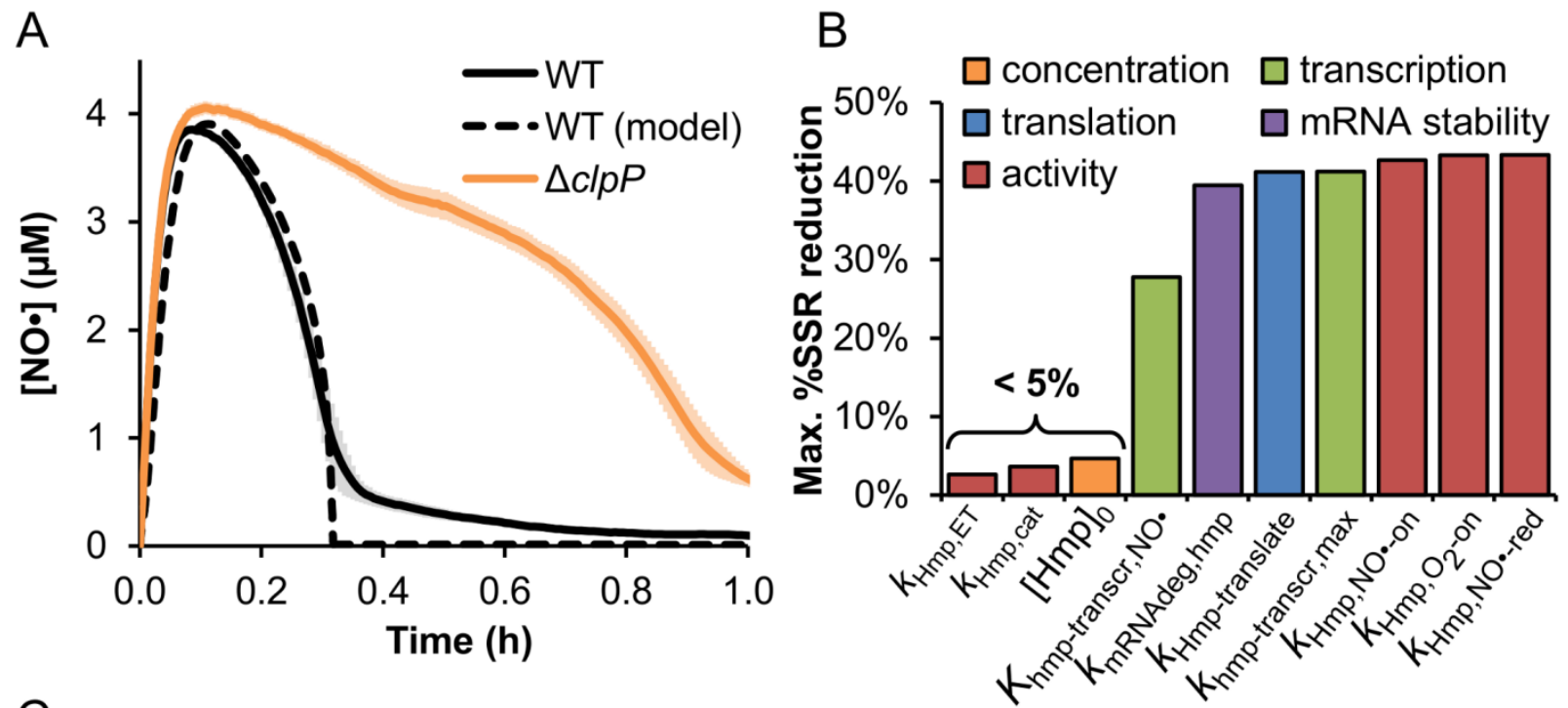

C

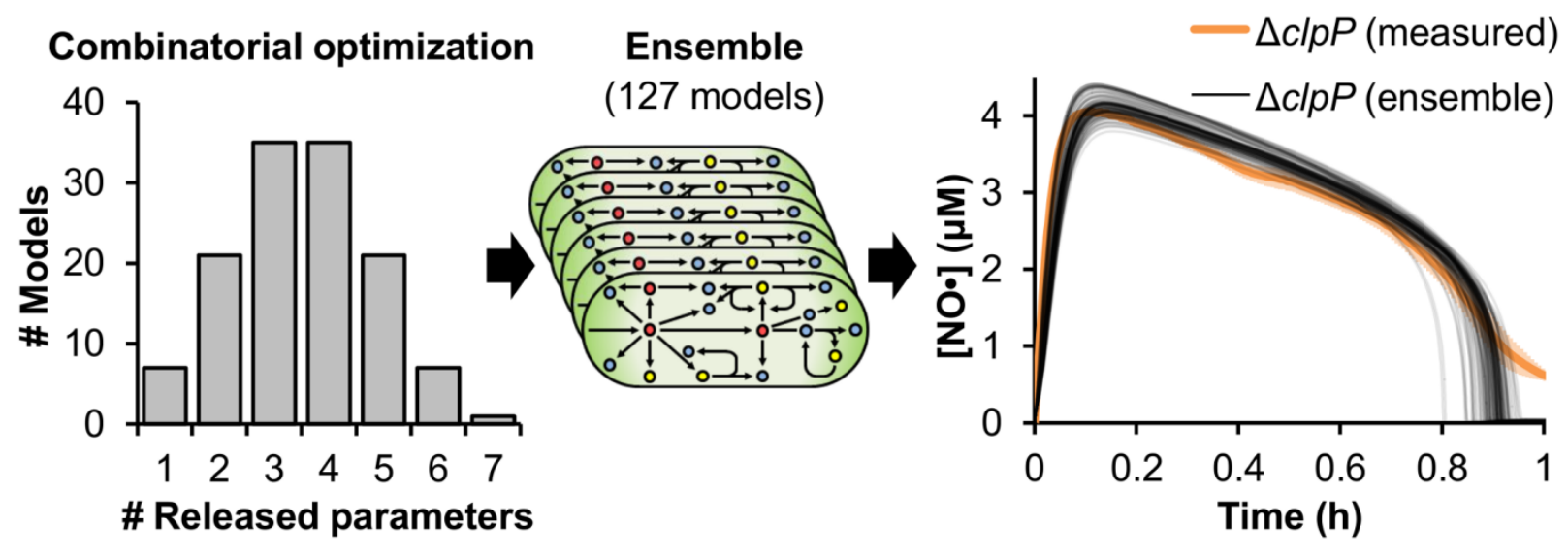

Figure 2. Generation of an ensemble of models that describe the NO•-sensitive phenotype of $\Delta \boldsymbol{c l p P}$. (A) Simulated [NO•] curve for WT E. coli (dashed black line) is shown along with the measured [NO•] curves (from Fig. $1 \mathrm{~B}$ ) for WT and $\Delta c l p P$ (solid black and orange lines, respectively) following DPTA NONOate treatment. Data are the mean of at least 3 independent experiments, with error bars representing the standard error of the mean. (B) Maximum reduction in error (SSR) between the simulated and measured $\Delta c l p P[\mathrm{NO} \bullet$ ] curve achieved upon individually varying each model parameter within two-fold of its original value. The top 10 parameters yielding the greatest decrease in SSR are shown, and are grouped in categories by color (orange, initial Hmp concentration; blue, Hmp translation; red, Hmp catalytic activity; green, $h m p$ transcription; purple, $h m p$ mRNA transcript stability). (C) Each of the 7 parameters from (B) exhibiting a $>5 \%$ decrease in SSR in were released and optimized (individually and combinatorially) to fit the measured $\Delta c l p P[\mathrm{NO} \bullet]$ curve, resulting in a total of 127 models. Shown on the far right are the [NO•] curves predicted by all 127 ensemble members superimposed on the measured $\Delta c l p P[\mathrm{NO} \bullet]$ curve from (A).

The analysis identified seven parameters whose adjustment could largely reduce the error (quantified as the sum of the squared residuals; SSR) between the model and $\Delta c l p P$ experimental 
data (Fig. 2B), as well as many parameters that could not. This information was useful because it allowed effort to be focused on elements likely to give rise to the $\Delta c l p P$ phenotype, rather than those that were likely to be negligible contributors. For example, it allowed us to disregard initial Hmp concentration and rate of enzyme degradation as potential explanations for the phenotype.

The seven parameters that could largely reconcile the model predictions with $\triangle c l p P$ data were all associated with Hmp, which was anticipated given the importance of the enzyme to $E$. coli $\mathrm{NO} \cdot$ detoxification under aerobic conditions. Three of the parameters governed the catalytic activity of $\mathrm{Hmp}\left(k_{\mathrm{Hmp}, \mathrm{O} 2-\mathrm{on}}, k_{\mathrm{Hmp}, \mathrm{NO} \cdot-\mathrm{on}}, k_{\mathrm{Hmp}, \mathrm{NO} \cdot-\mathrm{red}}\right)$, three its transcript levels $\left(k_{\mathrm{Hmp}-\text { transcr,max }}, K_{\mathrm{Hmp}-}\right.$ transcr,NO,$\left.k_{\text {mRNAdeg,hmp }}\right)$, and one its translation rate $\left(k_{\text {Hmp-translate }}\right)$. Changing any one of these parameters gave rise to different models, each representing a distinct mechanism through which $\triangle c l p P$ may be impairing the rate of $\mathrm{NO} \bullet$ detoxification. We reasoned that perturbations to the catalytic activity, mRNA synthesis, mRNA degradation, or translation of Hmp, as indicated by the seven parameters, could result from loss of ClpP. Hmp utilizes heme in its active site, and it is conceivable that the absence of ClpP could reduce the availability of heme during NO• stress. With regard to transcription, ClpP may be degrading a transcriptional regulator that inhibits $h m p$ expression. In the context of transcript stability, ClpP could be responsible for degradation of an RNase in the presence of $\mathrm{NO} \bullet$, thereby enhancing the lifetime of $h m p$ mRNA. With respect to translation, $\mathrm{NO} \bullet$ has previously been shown to result in amino acid limitation by damaging biosynthetic enzymes that contain iron-sulfur clusters (e.g., IlvD) [6,7]. Therefore, ClpP could promote translation under $\mathrm{NO} \bullet$ stress by providing a source of amino acids through the digestion of proteins. In addition, accumulation of misfolded or damaged proteins under NO• stress in $\triangle c l p P$ could have far-reaching effects on bacterial physiology. Lastly, we also recognized that the impact of $\triangle c l p P$ on $\mathrm{NO} \cdot$ detoxification may arise from a combination of these mechanisms. 
To account for this uncertainty and determine which cellular processes underlie the NO•sensitivity of $\Delta c l p P$, a systematic ensemble-guided approach was employed.

Analysis of an ensemble of plausible models allowed a systematic and exhaustive evaluation of the various physiological states that can describe the $\mathrm{NO} \cdot$ dynamics observed in $\Delta c l p P$ cultures. To generate the ensemble, the reaction network structure was maintained, but each of the seven parameters found to improve agreement between the model and $\Delta c l p P$ experimental data when varied were optimized individually and combinatorially (all combinations of $2,3,4,5,6$, and 7 parameters) to fit the $\Delta c l p P[\mathrm{NO} \bullet]$ curve. The parameters were allowed to vary within one order of magnitude of the WT value, yielding 127 distinct models that were each capable of accurately predicting the experimental data (Fig. 2C). This ensemble represented 127 potential mechanisms by which the $\Delta c l p P$ mutation could perturb the E. coli $\mathrm{NO} \bullet$ response network. To identify the correct mechanism, we performed in silico experiments with the ensemble to determine genetic and environmental perturbations that would allow discrimination between the ensemble members. The corresponding experiments were then performed, allowing reduction of the ensemble by removal of models that were inconsistent with the new data. This model discrimination process was iterated until convergence upon a single mechanism was achieved.

\subsubsection{Effect of $\Delta \mathrm{clpP}$ on Hmp catalytic activity}

Three of the parameters found to reconcile model simulations with $\triangle c l p P$ data govern the catalytic activity of Hmp. Ensemble members with modifications to these parameters predicted that Hmp in $\triangle c l p P$ was affected in such a way as to reduce its $\mathrm{NO} \bullet$ detoxification rate, yielding the observed slower NO• clearance than WT. To evaluate whether a reduction in Hmp catalytic activity was responsible for the impaired $\mathrm{NO} \bullet$ detoxification in $\triangle c l p P$, we rationalized that the 
enzyme could be pre-expressed prior to $\mathrm{NO} \bullet$ stress to achieve equal initial Hmp concentrations, and a translation inhibitor could be used to halt any additional protein synthesis during NO• treatment. These conditions were simulated by setting the initial concentration of Hmp to a value of $20 \mu \mathrm{M}$ (approximately one order of magnitude greater than the basal Hmp concentration predicted by the model), and deactivating translation. DPTA NONOate delivery was simulated to occur prior to the addition of cells, such that the $\mathrm{NO} \bullet$ could first accumulate and achieve a relatively stable concentration. We note that an NO•-containing environment was established prior to the addition of cells because the pre-expressed Hmp would not allow $\mathrm{NO} \bullet$ to reach appreciable concentrations if cells were added prior to DPTA NONOate dose. The delivery of $\mathrm{NO} \cdot$ to cell-free media was thus simulated for $10 \mathrm{~min}$, followed by an introduction of the cellular compartment (including $20 \mu \mathrm{M} \mathrm{Hmp}$ and disabled translation). The resulting [NO•] curves generated from the 127 different $\triangle c l p P$ models exhibited substantial variation, with NO• clearance times ranging from $1 \mathrm{~min}$ to $4.5 \mathrm{~h}$ after addition of cells, suggesting that an experiment would have considerable power to discriminate between models (Fig. 3A). As expected, members of the ensemble with altered Hmp catalytic activity predicted [NO•] curves that differed from the WT simulation, whereas the remaining ensemble predictions were identical to that of WT. 

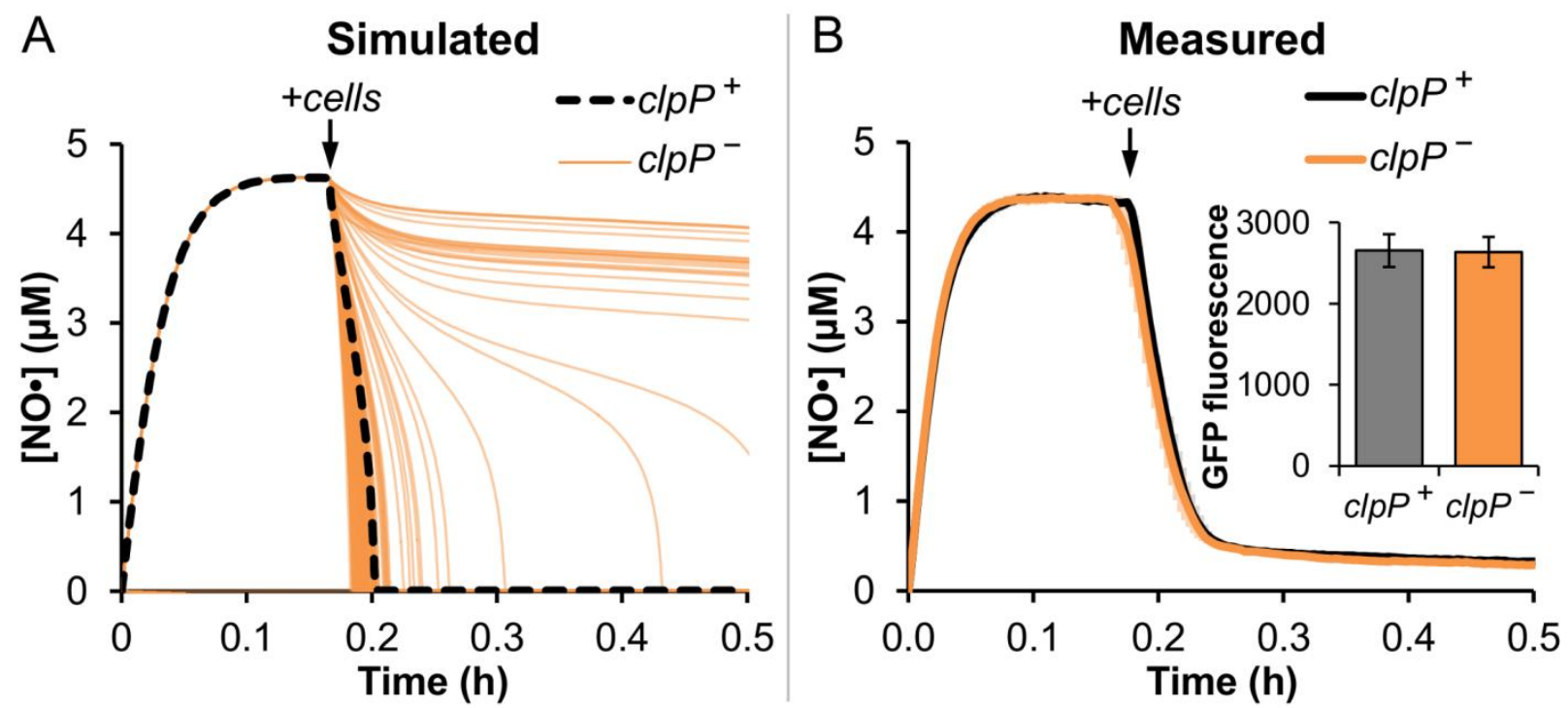

Figure 3. Hmp catalytic activity is unaffected by the $\Delta c l p P$ mutation. (A) Simulated and (B) experimentally measured [NO•] curves resulting from the addition of $c l p P^{+}$or $c l p P^{-}$cells containing equal concentrations of pre-expressed Hmp-GFP to NO•-treated media $(250 \mu \mathrm{M}$ DPTA NONOate) $10 \mathrm{~min}$ after dose, to a final $\mathrm{OD}_{600}$ of 0.05 . [NO•] curves in (A) were simulated by the $c l p P^{+}$model (dashed black line) and each of the 127 models of the $c l p P^{-}$ ensemble (thin orange lines), with the initial Hmp concentration set to $20 \mu \mathrm{M}$, and translation disabled. Pre-expression of Hmp-GFP for the experimental measurements in (B) was achieved by inducing expression from the pJR03 plasmid with $50 \mu \mathrm{M}$ IPTG for $15 \mathrm{~min}$, after which translation was inhibited with the addition of $50 \mu \mathrm{g} / \mathrm{ml} \mathrm{CAM}$. Fluorescence of the $\operatorname{clp} P^{+}$and $c l p P^{-}$cultures were measured at the time of cell delivery (shown in (B) inset), confirming equal concentrations of pre-expressed Hmp-GFP. Fluorescence of $c l p P^{+}$and $c l p P^{-}$containing the empty pQE-80L vector (no hmp-gfp) were subtracted as a blank. [NO•] curves and fluorescence readings in (B) are the mean of 3 independent experiments, with error bars representing the standard error of the mean.

To determine which members of the ensemble more accurately represented the $\Delta c l p P$ dynamics, the experiments analogous to the in silico conditions were conducted. hmp was cloned into the pQE-80L plasmid (Qiagen) to enable IPTG-inducible expression. Furthermore, to allow real-time quantification of Hmp levels within cells, and thus achieve identical concentrations between WT and $\Delta c l p P$, green fluorescent protein (GFP) was translationally fused to the Hmp Cterminus via a short peptide linker. The plasmid (pJR03) was transformed into $\Delta h m p$ and $\Delta h m p \Delta c l p P$ backgrounds (designated $c l p P^{+}$and $c l p P^{-}$, respectively), to eliminate participation of the chromosomal hmp. Expression of hmp-gfp was induced for 15 min with $50 \mu \mathrm{M}$ IPTG in 
exponential-phase cultures, followed by treatment with $50 \mu \mathrm{g} / \mathrm{ml}$ chloramphenicol (CAM) to halt further protein synthesis. Meanwhile, DPTA NONOate $(250 \mu \mathrm{M})$ was delivered to cell-free media (also containing $50 \mu \mathrm{g} / \mathrm{ml} \mathrm{CAM}$ ), and the $\mathrm{NO} \bullet$ concentration was allowed to stabilize for 10 min. At this time, $c l p P^{+}$or $c l p P^{-}$cells with pre-expressed Hmp-GFP were delivered to the NO•-treated media, and the resulting [NO•] was monitored (Fig. 3B). Fluorescence measurements at the time of cell delivery confirmed equal initial concentrations of Hmp-GFP for $c l p P^{+}$and clpP $P^{-}$cells. The NO• consumption rate of $c l p P^{+}$and $c l p P^{-}$cultures was indistinguishable, demonstrating that the $\triangle c l p P$ mutation had a negligible effect on the catalytic activity of Hmp. Controls run identically without IPTG induction, or with an empty pQE-80L vector (lacking the hmp-gfp coding sequence) were unable to clear $\mathrm{NO} \bullet$, confirming that the $\mathrm{NO} \bullet$ consumption was the result of Hmp-GFP expression (Fig. S2). Ensemble members involving a perturbation to Hmp catalytic parameters (112 models) were thus rejected, reducing the ensemble size to 15 models, all of which predicted that similar Hmp abundance will result in similar NO• clearance.

\subsubsection{Effect of $\Delta \mathrm{clpP}$ on gene expression from the hmp promoter}

The remaining ensemble members were models with differences in parameters governing Hmp translation and/or transcript abundance. To discriminate between these mechanisms, we simulated mRNA and protein levels from the $h m p$ promoter $\left(\mathrm{P}_{h m p}\right)$ driving $g f p$ expression. $h m p$ was removed from these simulations in order to maintain identical $\mathrm{NO} \bullet$ environments between WT and $\Delta c l p P$, and was replaced by $g f p$ to facilitate real-time quantification of protein levels in subsequent experiments. Delivery of $250 \mu \mathrm{M}$ DPTA NONOate was simulated for the $c l p P^{+}$ model and the clpP $P^{-}$ensemble, yielding predicted concentration profiles of $g f p$ mRNA and protein (Figs. 4A and 4B, respectively). The [mRNA] curves predicted by the clpP $P^{-}$ensemble all 
laid below that of $c l p P^{+}$, except for the $c l p P^{-}$model representing a mechanism described solely

by a deficiency in translation, which predicted [mRNA] equal to that of $c l p P^{+}$. The simulated

[GFP] curves were lower for all members of the ensemble relative to clp $P^{+}$.
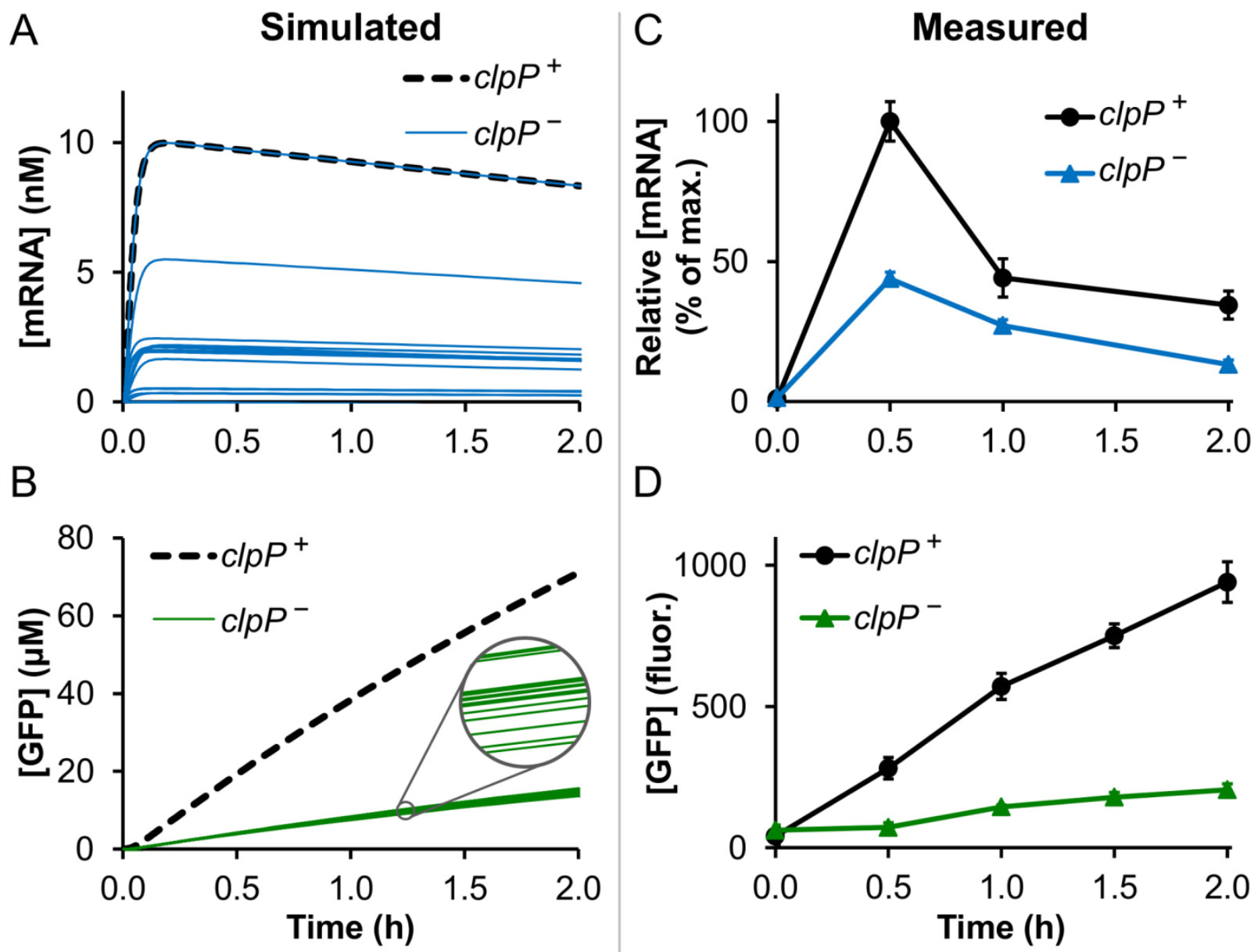

Figure 4. Deletion of $c l p P$ results in lower mRNA levels from $\mathbf{P}_{h m p}$ under NO・ stress. (A) $g f p$ mRNA concentrations and (B) associated GFP concentrations simulated by the $c l p P^{+}$model (black dashed line) and remaining $15 \mathrm{clp} P^{-}$ensemble members (thin blue and green lines for $g f p$ mRNA and GFP concentrations, respectively) resulting from the addition of $250 \mu \mathrm{M}$ DPTA NONOate to cells expressing GFP from the $h m p$ promoter $\left(\mathrm{P}_{h m p}\right)$. Hmp was disabled to maintain similar NO• environments between the $c l p P^{+}$and $c l p P^{-}$strains. Experimentally measured (C) gfp mRNA concentrations and (D) associated GFP concentrations (fluorescence) resulting from the addition of $250 \mu \mathrm{M}$ DPTA NONOate to $\Delta h m p$ and $\Delta h m p \Delta c l p P$ cells $\left(c l p P^{+}\right.$and $c l p P^{-}$, respectively) possessing the pJR04 plasmid, which contains $g f p$ under the control of the $\mathrm{P}_{h m p}$ promoter. Data are the mean of at least 3 independent experiments, with error bars representing the standard error of the mean. Fluorescence of $c l p P^{+}$and $c l p P^{-}$cells possessing the empty pUA66 plasmid (pJR04 without the $\mathrm{P}_{h m p}$ promoter) were subtracted as blanks in (D). 
Experimental investigation of the simulated condition was executed using a GFP reporter plasmid, where $g f p$ was placed under the control of the native $h m p$ promoter (pJR04). Activity of Hmp was eliminated by transforming pJR04 into $\Delta h m p$ and $\Delta h m p \Delta c l p P\left(\operatorname{clp} P^{+}\right.$and $c l p P^{-}$, respectively). Cultures of $c l p P^{+}$and $c l p P^{-}$were dosed with $250 \mu \mathrm{M}$ DPTA NONOate, and the production of $g f p$ mRNA and protein were measured with real-time quantitative PCR (qPCR) and fluorescence, respectively (Figs. 4C and 4D). The $g f p$ transcript levels in the $c l p P^{+}$culture were over two-fold higher than that of $c l p P^{-}$by $0.5 \mathrm{~h}$ after DPTA NONOate dose, and remained higher even after $2 \mathrm{~h}$. These data confirmed that a transcript defect was present, and thereby rejected a mechanism describing the $\triangle c l p P$ phenotype as solely a translational deficiency. Fluorescence measurements (Fig. 4D) showed a substantially higher rate of GFP production in $c l p P^{+}$cultures compared to $c l p P^{-}$, and were in qualitative agreement with the relative [GFP] predicted by the models. Similar levels of mRNA and GFP between the two strains at time zero (before NO treatment) demonstrated that $c l p P^{+/-}$phenotypic differences were confined to NO•stress conditions.

\subsubsection{Evaluation of whether a translational defect contributes to the $\Delta \mathrm{clpP}$ phenotype}

The remaining ensemble was comprised of models that attributed the $\triangle c l p P$ phenotype to differences in transcript-specific parameters, or those parameters in combination with an altered rate of Hmp translation. The previous experiment established that transcript abundance plays a role in the $\triangle c l p P$ mechanism, but the participation of translation effects remained unclear. To differentiate the contributions of lower transcript levels and perturbed translation on the impaired NO• clearance of $\triangle c l p P$, we simulated conditions of identical mRNA concentrations among the strains. Under these conditions, mechanisms involving translational perturbations would exhibit differences in protein concentrations and NO• clearance from WT. To simulate such conditions, 
the NO• dependency of hmp transcription was replaced with that of a synthetic inducer (IPTG), which we reasoned should allow similar $h m p$ transcript levels to be achieved in $c l p P^{+}$and $c l p P^{-}$ cultures (Figs. S3A-C). However, upon conducting the corresponding experimental measurements, the $c l p P^{-}$culture was unable to achieve hmp-gfp transcript levels comparable to those of $c l p P^{+}$, despite full induction (1 mM IPTG) from an orthogonal promoter (Text S1, Figs. S3D-F), which suggested that the transcript deficiency was not localized to hmp and its promoter. Given this phenomenon, modeling of $h m p$ transcription was maintained as a function of IPTG concentration, but the assumption of identical transcription kinetics was eliminated. As an alternative approach to determine whether a translational defect was contributing to the $\Delta c l p P$ phenotype, we leveraged the fact that the protein (Hmp-GFP) concentration could be fluorometrically quantified in real-time. Thus, the IPTG concentration was reduced in the $c l p P^{+}$ model until its [Hmp-GFP] dynamics aligned with each member of the $c l p P^{-}$ensemble members, and the associated [NO•] and [mRNA] were simulated. Mechanisms consisting of only transcript-related effects predicted mRNA levels similar to that of $c l p P^{+}$(Figs. 5A-C), whereas those with perturbations to both transcription and translation predicted mRNA levels different from that of $c l p P^{+}$(Figs. 5D-F). 

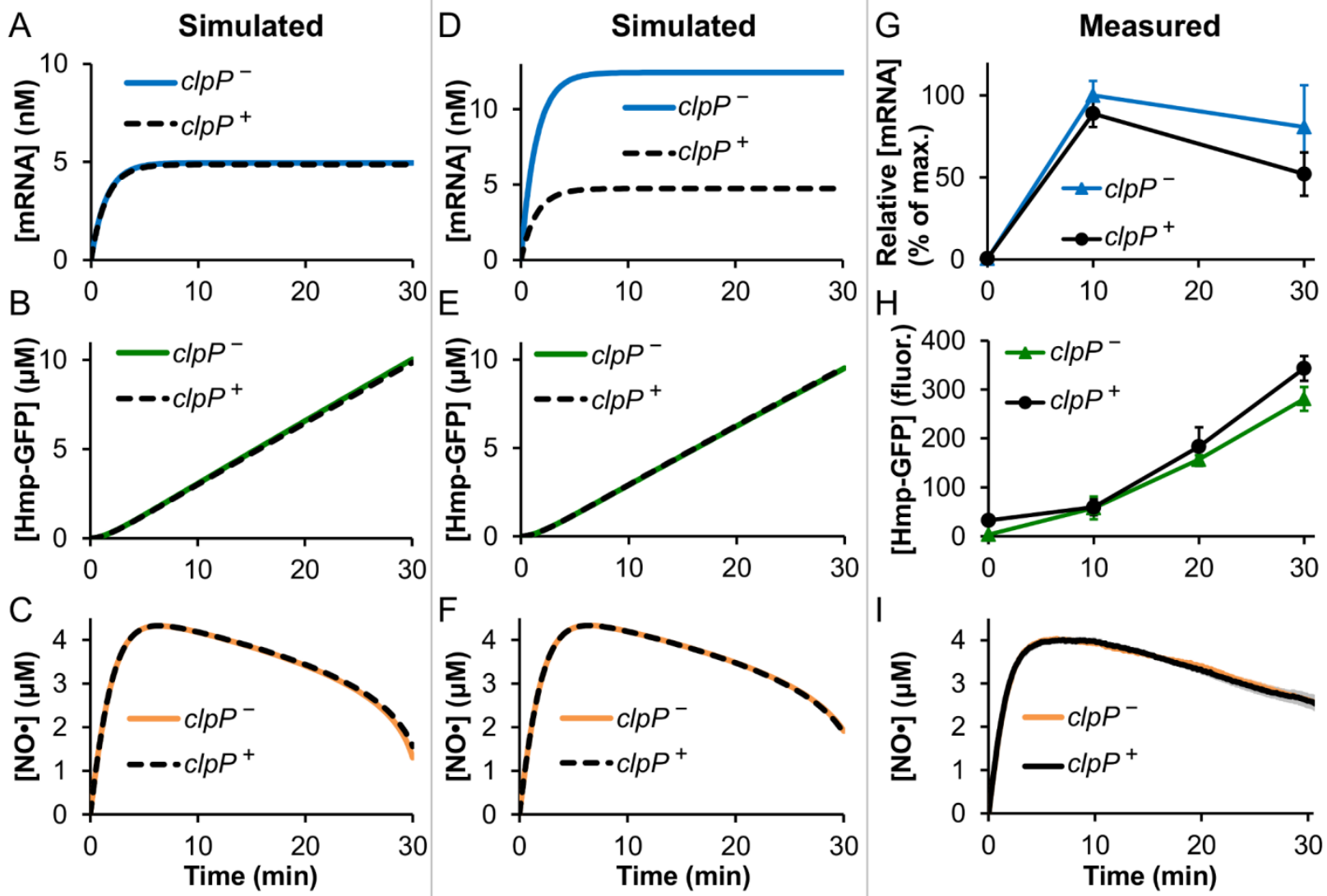

Figure 5. $\triangle$ clpP does not impair NO• detoxification through an impact on translation.

Shown are (A) hmp-gfp mRNA, (B) Hmp-GFP protein, and (C) NO• concentrations simulated by the $c l p P^{+}$model (black dashed lines) and a representative member of the remaining $c l p P^{-}$ ensemble (solid blue, green, and orange line for mRNA, protein, and NO•, respectively) with a perturbation to only a transcript-related parameter $\left(k_{\mathrm{hmp} \text {-transcr,max }}\right)$, resulting from the simultaneous addition of IPTG and $250 \mu \mathrm{M}$ DPTA NONOate to cells expressing $h m p$ - $g f p$ from an IPTG-inducible promoter. The IPTG concentration added to $c l p P^{+}$was optimized to obtain similar Hmp-GFP protein concentrations to that of the representative $c l p P^{-}$model (for which 1 mM IPTG was used). Simulation conditions in (D), (E), and (F) are identical to (A), (B), and (C), respectively, except using a different $c l p P^{-}$model, which was representative of ensemble members with perturbations to both translation- and transcript-related parameters $\left(k_{\mathrm{Hmp} \text {-translate }}\right.$ and $\left.k_{\text {hmp-transcr,max }}\right)$. IPTG added to $c l p P^{+}$cells was again optimized to obtain similar Hmp-GFP protein concentrations to that of the $c l p P^{-}$model. Representative ensemble members are shown here rather than all remaining $c l p P^{-}$models because for each $c l p P^{-}$model the corresponding $\operatorname{clp} P^{+}$simulation is different, due to tuning of [IPTG] to achieve [Hmp-GFP] identical to that in the $c l p P^{-}$model (see Fig. S4 for results from remaining ensemble members). Experimental measurements of (G) hmp-gfp mRNA, (H) Hmp-GFP protein, and (I) NO• concentrations resulting from the simultaneous addition of IPTG and $250 \mu \mathrm{M}$ DPTA NONOate to $\Delta h m p$ and $\Delta h m p \Delta c l p P$ cells $\left(c l p P^{+}\right.$and $c l p P^{-}$, respectively) possessing the pJR05 plasmid, which contains IPTG-inducible $\mathrm{P}_{\mathrm{T} 5}-h m p-g f p$. $c l p P^{-}$was fully-induced (1 mM IPTG), while $c l p P^{+}$was induced with $10 \mu \mathrm{M}$ IPTG to yield similar Hmp-GFP concentrations (fluorescence) to that of $c l p P^{-}$, consistent with the simulated conditions. Experimental data are the mean of at least 3 independent experiments, with error bars representing the standard error of the mean. [mRNA] is relative, presented as a percentage of the maximum concentration measured ( $c l p P^{-}$at $\left.10 \mathrm{~min}\right)$. 
Fluorescence readings of $c l p P^{+}$and $c l p P^{-}$possessing pJR05 without induction (no IPTG) were subtracted as blanks.

The associated experiments were performed using an IPTG-inducible hmp-gfp expression system (pJR05). The plasmid was transformed into $\Delta h m p$ and $\Delta h m p \Delta c l p P$ backgrounds $\left(c l p P^{+}\right.$ and $c l p P^{-}$, respectively), to eliminate contributing effects of the chromosomal hmp. Cultures of $c l p P^{+}$and $c l p P^{-}$were treated with $250 \mu \mathrm{M}$ DPTA NONOate, and immediately induced with IPTG, and the resulting [NO•] curve, GFP fluorescence, and transcript levels were quantified (Figs. 5G-I). In accordance with the in silico analysis, $c l p P^{-}$was fully induced (1 mM IPTG), while the induction of $c l p P^{+}$was reduced until a rate of Hmp-GFP production similar to that of $c l p P^{-}$was achieved (10 $\mu \mathrm{M}$ IPTG). Consistent with previous results (Fig. 3), similar protein levels resulted in similar NO• clearance between the two strains, leaving model discrimination to rely on whether a difference in mRNA levels was observed. The associated mRNA concentrations were quantified, and did not differ significantly between the two strains $(p>0.05$ for all points; two-tailed $t$-test with unequal variance), supporting mechanisms that described the $\Delta c l p P$ phenotype as only involving a perturbation to transcript levels. Mechanisms yielding different transcript levels for similar protein concentrations were therefore eliminated from the ensemble, reducing the size from 14 to 4 models (Fig. S4).

\subsubsection{Evaluation of contributions from transcript degradation}

The remaining ensemble included mechanisms attributing the $\mathrm{NO} \bullet$-sensitivity of $\Delta c l p P$ to attenuated mRNA production, increased mRNA degradation, or some combination of the two. To differentiate between these mechanisms, an in silico experiment was conducted wherein the cells were initialized with a nonzero $g f p$ mRNA concentration $(100 \mathrm{nM})$, but transcription was inactivated during $\mathrm{NO} \bullet$ stress, leaving mRNA degradation as the sole process dictating transcript 
concentration. $g f p$ mRNA was chosen to replace that of $h m p$ to maintain identical NO• environments between the $\operatorname{clp} P^{+}$and $c l p P^{-}$models, while also facilitating fluorometric quantification of protein abundance in subsequent experimental measurements. Treatment of cultures with $250 \mu \mathrm{M}$ DPTA NONOate was simulated, yielding different [GFP] profiles for each of the $c l p P^{-}$models (Fig. 6A).
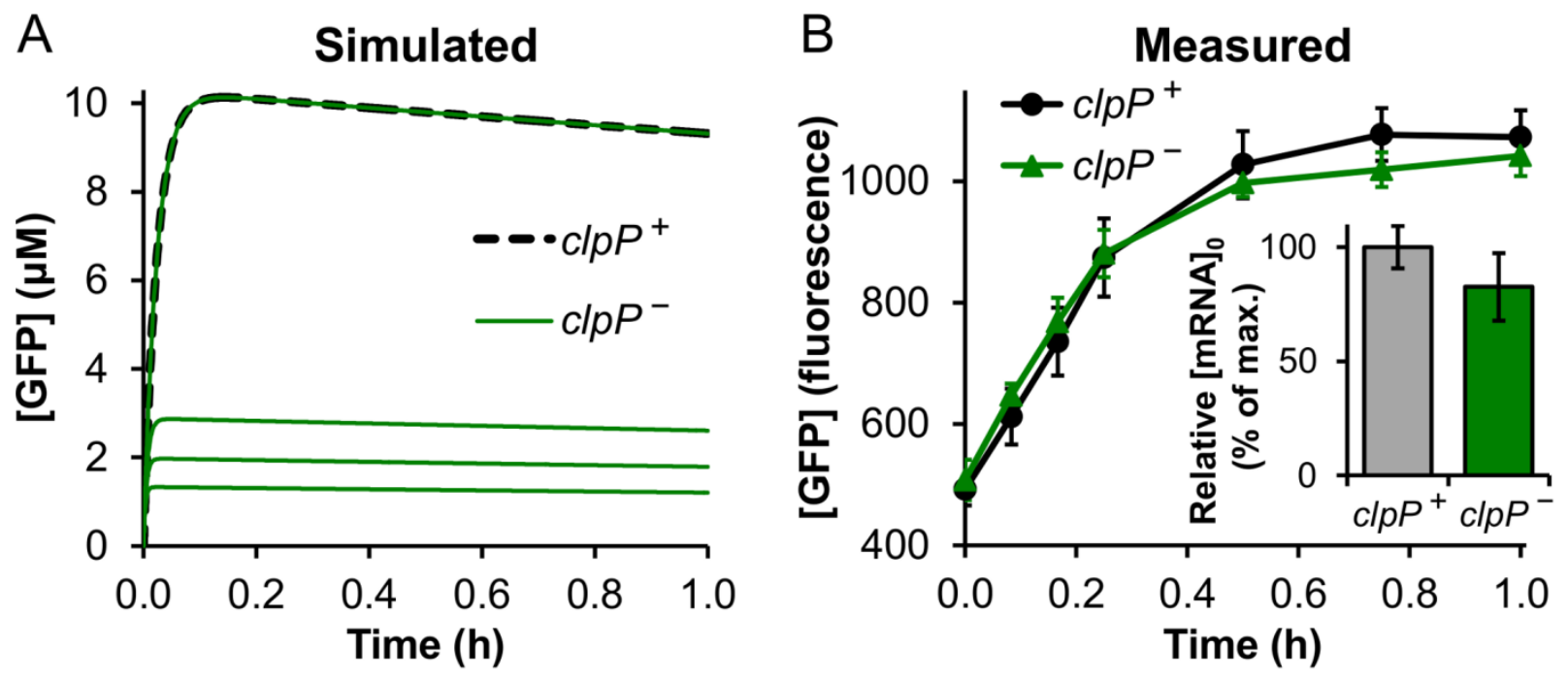

Figure 6. Nonspecific mRNA degradation is not increased in $\Delta c l p P$. (A) GFP concentrations simulated by the $c l p P^{+}$model (black dashed line) and the remaining four $c l p P^{-}$ensemble members (thin green lines) with an initial $g f p$ mRNA concentration set to $100 \mathrm{nM}$, transcription deactivated, and $250 \mu \mathrm{M}$ DPTA NONOate treatment. (B) Experimentally measured GFP levels (fluorescence) following treatment of $c l p P^{+}$and $c l p P^{-}$strains (possessing pJR06) with $500 \mu \mathrm{g} / \mathrm{ml}$ RIF to halt transcription, and $250 \mu \mathrm{M}$ DPTA NONOate. Cells were induced with $1 \mathrm{mM}$ IPTG for 15 min prior to treatment to express $g f p$ from the pJR06 plasmid, to establish a nonzero initial $g f p$ transcript concentration, which was confirmed (via qPCR) to be similar between $c l p P^{+}$and $\operatorname{clp} P^{-}$at the time of treatment (inset of $\mathbf{B}$ ). Fluorescence values of identically treated cells, but without induction, were subtracted as background from the values presented in (B).

Experimentally, the simulated conditions were reproduced using $\Delta h m p$ and $\Delta h m p \Delta c l p P$ cells transformed with a plasmid harboring IPTG-inducible $g f p$ (pJR06). Exponential-phase cultures were induced with $1 \mathrm{mM}$ IPTG for 15 minutes, washed and delivered to a bioreactor containing fresh media with $500 \mu \mathrm{g} / \mathrm{ml}$ rifampicin (RIF) to halt transcription, and immediately dosed with $250 \mu \mathrm{M}$ DPTA NONOate. To confirm that transcription was arrested upon treatment, 
the assay was repeated identically, except the IPTG was added immediately after RIF treatment, rather than 15 minutes prior (Fig. S5). The concentration of GFP was quantified with fluorescence readings, and $500 \mu \mathrm{L}$ of culture was removed at time zero (immediately prior to treatment) to confirm similar starting concentrations of $g f p$ mRNA using qPCR. The resulting [GFP] profile was similar in shape to that predicted by the model, with an increasing concentration that plateaued as mRNA was depleted, and the data revealed a similar rate of mRNA degradation in $c l p P^{+}$and $c l p P^{-}$(Fig. 6B). This reduced the ensemble to a single mechanism that attributed the $\mathrm{NO} \bullet$-sensitive phenotype of $\Delta c l p P$ to be the result of attenuated hmp transcription.

\subsection{Assessing the breadth of the $\Delta c l p P$ transcription defect}

\subsubsection{Investigation of an orthogonal expression system}

The ensemble modeling approach narrowed the source of the impaired NO• detoxification activity of $\Delta c l p P$ to an inability to achieve WT levels of $h m p$ mRNA transcripts. Interestingly, the presence of this effect during the ensemble dissection experiments utilizing an independent, orthogonal promoter $\left(\mathrm{P}_{\mathrm{T} 5}\right)$ suggested that the impact of $\Delta c l p P$ on transcript levels under NO• stress may be widespread. To further investigate the breadth of the $\triangle c l p P$ phenotype, a completely independent expression system was used, where the IPTG-inducible $\mathrm{P}_{\mathrm{T} 5}$ promoter was replaced with an anhydrotetracycline (aTc)-inducible $\mathrm{P}_{\mathrm{LtetO}-1}$, and GFP replaced with mCherry (pJR07). This alternate plasmid was transformed into $\Delta h m p$ and $\Delta h m p \Delta c l p P$ backgrounds (each possessing a $\mathrm{P}_{\mathrm{N} 25}$-tetR chromosomal knock-in) for comparison of mCherry transcript and protein production under identical NO• environments. Expression of mCherry was induced with the addition of $50 \mathrm{ng} / \mathrm{ml} \mathrm{aTc}$, and protein concentration was monitored 
fluorometrically. In the absence of $\mathrm{NO} \bullet$ stress, the $c l p P^{+}$and $c l p P^{-}$cultures produced similar concentrations of mCherry (Fig. S6A). However, upon treatment with $250 \mu \mathrm{M}$ DPTA NONOate, the $c l p P^{-}$cells exhibited significantly lower concentrations of mCherry than $c l p P^{+}$, an effect that was again reflected in the associated mRNA concentrations (Figs. S6B and S6C). The observation of this transcription defect for an entirely different promoter, inducer, mRNA, and protein was supportive of a widespread effect of $\triangle c l p P$ on transcript levels. To explore this effect further, a transcriptomic investigation was conducted using RNA-Seq.

\subsubsection{RNA-Seq analysis of NO stress in WT and $\Delta$ clpP}

Cultures of WT and $\triangle c l p P$ were grown to exponential phase and treated with $250 \mu \mathrm{M}$ DPTA NONOate (or left untreated for controls) under conditions identical to those of the original [NO•] curve measurements (Fig. 1B). The sampling time for subsequent RNA extraction was 10 min after DPTA NONOate delivery, as the [NO•] was relatively similar between WT and $\triangle c l p P$ at this time, and was just beginning to diverge (Fig. 1B). RNA was extracted and purified from WT and $\triangle c l p P$ cells, and further processed to yield mRNA (see Materials and methods). The mRNA was analyzed using RNA-Seq, and the results were evaluated for differences in transcript levels between untreated and NO•-treated samples, and between WT and $\Delta c l p P$.

In agreement with previous transcriptomic analyses of NO•-stressed E. coli under aerobic conditions [6,31], genes exhibiting a large expression increase for WT in response to NO• included those related to detoxification (norVW, $h m p$, and $h c p$ ), repair (ytfE), and respiration $(c y d A B)$. Analogously, in agreement with previous comparisons of $\triangle c l p P$ and WT under unstressed conditions, genes involved in the production of curli (csg genes) and fimbriae ( $\mathrm{fim}$ genes) were largely reduced in $\triangle c l p P$ due to repression by the flagellar system [32]. 
Comparison of mRNA counts between $\mathrm{NO} \bullet$-treated WT and $\Delta c l p P$ revealed a large effect of the $\Delta c l p P$ mutation on the NO• response. Of the 284 genes exhibiting a significant $(p<0.05)$ change in transcript levels of at least 10-fold (increase or decrease) in response to NO• in WT or $\Delta c l p P$, over a quarter (80 genes, $28.2 \%$ ) were identified as having significantly different transcript levels between NO•-treated WT and $\Delta c l p P$ (Fig. S7). One of the genes falling into this category was $h m p$, where transcript levels between WT and $\Delta c l p P$ did not differ significantly in the absence of $\mathrm{NO} \bullet$ stress, but were significantly higher in WT compared to $\triangle c l p P$ after $\mathrm{NO} \bullet$ treatment, confirming our previous qPCR analyses that indicated a decreased abundance of $h m p$ transcripts in NO•-stressed $\triangle c l p P$. The differentially expressed genes spanned a broad spectrum of cellular functions beyond $\mathrm{NO} \bullet$ defense, ranging from iron metabolism to purine biosynthesis (Table S1).

Given the observed perturbation to hmp mRNA levels, we considered the possibility that the $\triangle c l p P$ mutation was affecting one or more of the transcription factors associated with the hmp promoter (NsrR, FNR, Fur, or MetR) [33]. We found that the mRNA counts of the regulators themselves were not significantly different between WT and $\Delta c l p P$, either in the presence or absence of $\mathrm{NO} \cdot$. We next inspected the regulons of the transcription factors by considering genes without significant differences between WT and $\Delta c l p P$ levels prior to treatment that exhibited significant, $\geq 10$-fold changes in transcript levels in WT upon treatment with NO• Although significant differences in mRNA levels were observed between NO•-treated WT and $\Delta c l p P$ for genes belonging to each regulon, approximately half of the genes for each regulon were not significantly different between $\mathrm{WT}$ and $\Delta c l p P$, and those that differed showed a mix of heightened and attenuated responses (Table S2). This suggested that that effect of $\Delta c l p P$ 
cannot be pinpointed to a single transcription factor acting on the hmp promoter, but instead affects transcription more broadly.

\subsection{Identification of interaction partners of ClpP that contribute to its effect on the NO•} response network

Upon confirming that $\mathrm{ClpP}$ was exerting a global effect on transcript levels under NO• stress, we sought to determine how ClpP, a protease, exerts this control. ClpP is a serine protease which targets a large number of substrates in the cell, ranging from SsrA-tagged proteins to sigma factors, with help from its ATPase adapter (unfoldase) subunits ClpA and ClpX [22]. Therefore, deletion mutants lacking either ClpA, ClpX, or both ClpA and ClpX, were evaluated for their ability to resume growth following NO• stress and to detoxify NO• (Fig. 7).
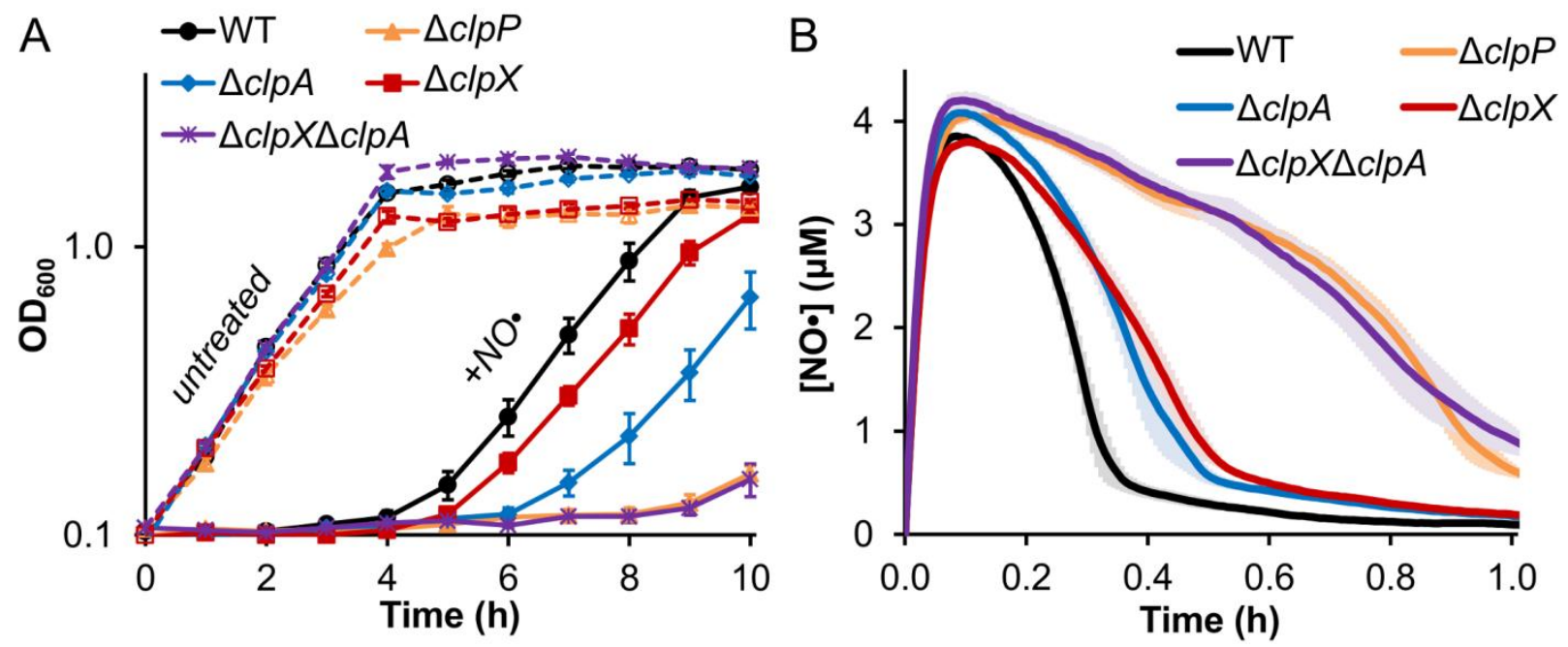

Figure 7. ClpA and ClpX are required for the NO-protective effect of ClpP. (A) Cultures were grown aerobically to exponential phase, diluted to an $\mathrm{OD}_{600}$ of 0.1 , and treated with $2 \mathrm{mM}$ DPTA NONOate (solid lines, filled markers), or left untreated (dashed lines, open markers), and growth was monitored hourly for $10 \mathrm{~h}$ post-dose. (B) Measured [NO•] profiles resulting from the addition of $250 \mu \mathrm{M}$ DPTA NONOate to aerobic, exponential-phase cultures at an $\mathrm{OD}_{600}$ of 0.05 . The data are the mean of at least 3 independent experiments, with error bars representing the standard error of the mean. 
Relative to WT, the $\Delta c \operatorname{lp} A$ and $\Delta c l p X$ mutants exhibited a 1 to $2 \mathrm{~h}$ longer delay in growth resumption after $\mathrm{NO} \bullet$ stress and a slower rate of $\mathrm{NO} \bullet$ detoxification $(\sim 40 \%$ increase in clearance time), but the effect was attenuated relative to that of $\Delta c l p P$. However, the double mutant lacking both $\mathrm{ClpP}$ adapters $(\triangle \operatorname{clp} A \Delta \operatorname{clp} X)$ reproduced the impaired growth and inhibited NO• detoxification phenotype of $\Delta c l p P$. This demonstrated that the ClpP proteolytic subunit alone is insufficient to confer a $\mathrm{NO} \bullet$-protective effect, and it requires both the ClpA and ClpX adapters.

To assess the involvement of known ClpAP and ClpXP substrates and interaction partners in the $\triangle c l p P \mathrm{NO} \bullet$-sensitive phenotype, $\mathrm{NO} \bullet$ consumption was measured for numerous deletion mutants in WT and $\Delta c l p P$ backgrounds, focusing on those with potential to broadly impact transcript levels or cellular function. Interestingly, trans-translation (SsrA, SspB), Dps, RpoS, RssB, Rsd, and ClpS were all found to not participate in the NO• detoxification phenotype of $\Delta c l p P($ Text S1 and Fig. S8).

Collectively, these data demonstrate that the role of $\mathrm{ClpP}$ in regulating transcript levels under $\mathrm{NO} \bullet$ stress requires ClpA and ClpX activity. Although the substrates degraded by ClpP to promote $\mathrm{NO} \bullet$ detoxification are beyond the known ClpP degradation targets that broadly impact cellular physiology and/or transcription, or are a combination of multiple regulatory systems, the data presented here convincingly demonstrate that inhibiting ClpP protease would be an effective means to potentiate $\mathrm{NO} \cdot$ toxicity in E. coli.

\section{Discussion}

Antibiotic-resistant infections continue to increase in frequency and severity while the supply of new antibiotics continues to diminish [34-36]. To address this growing therapeutic gap, alternative anti-infective approaches, such as antivirulence therapies, are being explored [37,38]. 
Unlike traditional antibiotics, which target essential cellular processes, antivirulence therapies are designed to disrupt critical host-pathogen interactions. This focuses selective pressure to the host environment during the infection process - the time and place it is needed - which minimizes the driving force for resistance development [39]. Furthermore, antivirulence treatments are less likely to deleteriously affect commensal bacteria, which is an unfavorable side effect of many traditional antibiotics that can lead to complications including secondary infections by opportunistic pathogens, such as Clostridium difficile [40]. Given the strong influence of the bacterial $\mathrm{NO} \cdot$ defense network on virulence for a broad range of pathogens, this network is a prime target for antivirulence therapies. Unfortunately, the major NO• detoxification systems, such as $\mathrm{NO} \bullet$ dioxygenase and $\mathrm{NO} \bullet$ reductase, have proven to be difficult to drug due to the host toxicity and poor transport into bacterial cells of known inhibitors (e.g., cyanide [20] and imidazole [21]). Therefore, the identification of additional targets that can be leveraged to disable bacterial $\mathrm{NO} \bullet$ defense systems will facilitate the development of antivirulence treatments.

Here, we identified ClpP and its ATPase adapters ClpA and ClpX as a critical mediators of the E. coli NO• stress response. Deletion of ClpP or both ClpA and ClpX crippled the ability of $E$. coli to detoxify $\mathrm{NO} \bullet$ from the environment, thereby prolonging bacteriostasis and enhancing the damaging effects of $\mathrm{NO} \cdot$. Furthermore, the impaired $\mathrm{NO} \bullet$ detoxification caused by the loss of ClpP was shown to translate to pathogenic E. coli $(\mathrm{O} 157: \mathrm{H} 7)$. This data suggests that ClpP protease activity may be an effective target for antivirulence therapies against pathogenic E. coli if effective inhibitors can be found. Interestingly, the importance of $\mathrm{ClpP}$ to the pathogenesis of a number of species including L. monocytogenes [27], S. pneumoniae [24], S. aureus [41], and EHEC [42] has already prompted the search for inhibitor compounds [43,44]. 
Bacterial protease activity has previously been found to contribute to the $\mathrm{NO} \cdot$ defenses of other pathogens [10,24]. For example, M. tuberculosis mutants lacking core subunits of the proteasome displayed enhanced sensitivity to acidified nitrite [10], and an iNOS inhibitor improved the viability of a $S$. pneumoniae $\Delta c l p P$ mutant that exhibited decreased survival within untreated macrophages [24]. However, the mechanism bridging protease activity to NO• defense in both of these studies was not explored, though it was speculated that the protease could be responsible for digesting NO•-damaged proteins that may otherwise accumulate to toxic levels within the cell [10]. In this work, not only did we identify a novel NO•-defensive role for ClpP in E. coli, but we investigated the underlying mechanism and found $\mathrm{ClpP}$ to be a global regulator of transcript levels under $\mathrm{NO} \bullet$ stress in E. coli, an effect of which was to promote $\mathrm{NO} \bullet$ detoxification through increased levels of $h m p$ mRNA. This discovery was facilitated by an ensemble-guided approach, where a collection of 127 models was generated to capture the [NO•] dynamics of $\triangle c l p P$, and a model discrimination procedure was iterated until a single model remained, which attributed the increased $\mathrm{NO} \bullet$ sensitivity of $\Delta c l p P$ to reduced transcription of hmp under NO• stress. This approach is especially useful for analyzing phenotypes involving broadly reactive molecules such as $\mathrm{NO} \bullet$, for which the biochemistry is dictated by complex networks of reactions and interactions.

Ensemble modeling is a methodology that has previously been employed to computationally explore systems for which uncertainty exists in parameter values and/or network structure [45-56]. For example, Wang and Hatzimanikatis used a Monte Carlo method to address kinetic parameter uncertainty in a metabolic control analysis (MCA) study of Saccharomyces cerevisiae central carbon metabolism, and identified major differences in control schemes between batch and chemostat culturing conditions [46]. Measured concentrations of metabolites 
involved in the $S$. cerevisiae trehalose pathway were used by Jia and colleagues to generate an ensemble of models, from which randomly-selected models were shown to capture the data equally well [51]. Tran and colleagues demonstrated how an ensemble of $E$. coli central metabolism models with known steady-state parameters, but unknown kinetics, could be filtered using a model discrimination process, and ultimately used to predict perturbation phenotypes [48]. The target-of-rapamycin (TOR) pathway of S. cerevisiae was studied by Keupfer and colleagues using an ensemble of nineteen different possible network structures, where guided experiments were used to reduce the ensemble, revealing the importance of the Tap42p-Tip41p complex formation in signal control [47].

The study described here demonstrates yet another application of an ensemble-guided approach, complementing the growing body of literature on the use of ensemble modeling to analyze biochemical networks. We anticipate that ClpP may constitute an effective antivirulence target, helping to mitigate the mounting crisis of antibiotic resistance, and that the ensembleguided approach will prove useful for studying NO• stress in different conditions and pathogens, as well as other broadly reactive immune antimicrobials such as $\mathrm{H}_{2} \mathrm{O}_{2}$.

\section{Materials and methods}

\subsection{Bacterial strains and plasmids}

The bacteria used in this study were E. coli K-12 MG1655 and enterohemorrhagic E. coli O157:H7 TUV93-0. All mutants and transformants were derived from one of these two parent strains, and are described in Table S3. 


\subsection{Bioreactor}

To allow continuous [NO•] measurements in a growing culture, all assays (unless stated otherwise) were conducted in a stirred batch bioreactor, identical to our previous studies [29,30]. The bioreactor consisted of a disposable $50 \mathrm{~mL}$ conical tube (Falcon) suspended in a $37^{\circ} \mathrm{C}$ stirred water bath, and it contained $10 \mathrm{~mL}$ of growth media. The culture was continuously stirred with a 0.5 in. magnetic stir bar, and was open to ambient air.

\subsection{NO• measurements and chemicals}

[NO•] was measured continuously (20 samples/s) using an ISO-NOP $2 \mathrm{~mm} \mathrm{NO \bullet} \mathrm{sensor}$ (World Precision Instruments). Sensor calibration was performed daily according to the manufacturer's instructions. Briefly, various concentrations of an $\mathrm{NO}_{2}{ }^{-}$standard (Fisher) were delivered to a solution of $0.1 \mathrm{M} \mathrm{KI}+0.1 \mathrm{M} \mathrm{H}_{2} \mathrm{SO}_{4}$ (producing $\mathrm{NO} \cdot$ from $\mathrm{NO}_{2}^{-}$in a 1:1 molar ratio) in the bioreactor to generate a calibration curve, which allowed conversion of the output signal (pA) to $\mathrm{NO} \bullet$ concentration $(\mu \mathrm{M})$. NO・ was delivered to cultures using the chemical donor, DPTA NONOate ((Z)-1-[N-(3-aminopropyl)- $N$-(3-ammoniopropyl)amino]diazen-1-ium-1,2diolate) (Cayman Chemical), which dissociates to release 2 molecules of $\mathrm{NO} \cdot$ per parent compound.

\subsection{Absorbance and fluorescence measurements}

Cell concentration (optical density at $\left.600 \mathrm{~nm} ; \mathrm{OD}_{600}\right)$ and fluorescence $(485 / 515 \mathrm{~nm}$ excitation/emission for GFP, or 580/610 nm excitation/emission for mCherry) were quantified using a Synergy ${ }^{\mathrm{TM}} \mathrm{H} 1 \mathrm{Hybrid}$ Microplate Reader. 


\subsection{NO•-stressed growth assay}

E. coli were grown aerobically from a $-80^{\circ} \mathrm{C}$ frozen stock in $1 \mathrm{ml} \mathrm{LB}$ at $37^{\circ} \mathrm{C}$ and 250 r.p.m. for $4 \mathrm{~h} .10 \mu \mathrm{L}$ of the LB pre-culture were used to inoculate $1 \mathrm{ml}$ of MOPS minimal media (Teknova) with $10 \mathrm{mM}$ glucose, which was grown overnight $(16 \mathrm{~h})$ at $37^{\circ} \mathrm{C}$ and 250 r.p.m. The overnight culture was inoculated into $2 \mathrm{ml}$ of fresh MOPS + glucose to an $\mathrm{OD}_{600}$ of 0.01 , and grown until mid-exponential phase $\left(\mathrm{OD}_{600}=0.2\right)$. The exponential-phase culture was quickly transferred to two separate test tubes and diluted with fresh, pre-warmed $\left(37^{\circ} \mathrm{C}\right) \mathrm{MOPS}+$ glucose to achieve identical $1 \mathrm{ml}$ cultures at an $\mathrm{OD}_{600}$ of 0.1 . One culture was dosed immediately with 2 mM DPTA NONOate, while the other was treated only with the DPTA NONOate solvent $(0.14 \mathrm{mM}$ final $[\mathrm{NaOH}])$. Both cultures were grown at $37^{\circ} \mathrm{C}$ and 250 r.p.m. for $10 \mathrm{~h}$, with $\mathrm{OD}_{600}$ measured hourly.

\subsection{NO• consumption assay}

E. coli from a $-80^{\circ} \mathrm{C}$ frozen stock were grown aerobically in $\mathrm{LB}$ for $4 \mathrm{~h}$, and MOPS minimal media with $10 \mathrm{mM}$ glucose for $16 \mathrm{~h}\left(37^{\circ} \mathrm{C}\right.$ and 250 r.p.m. $)$. The overnight culture was inoculated into $20 \mathrm{ml}$ of fresh MOPS + glucose media in a $250 \mathrm{ml}$ baffled shake flask to an initial $\mathrm{OD}_{600}$ of 0.01 , and grown at $37^{\circ} \mathrm{C}$ and 250 r.p.m. until $\mathrm{OD}_{600}=0.2$. The culture was centrifuged for 3 min at 15,000 r.p.m., resuspended in fresh pre-warmed $\left(37^{\circ} \mathrm{C}\right) \mathrm{MOPS}+$ glucose, centrifuged for an additional 3 min at 15,000 r.p.m., and again resuspended in pre-

warmed MOPS + glucose. The resuspension was delivered to the bioreactor to a final $\mathrm{OD}_{600}$ of 0.05, and dosed immediately with $250 \mu \mathrm{M}$ DPTA NONOate. 


\subsection{Hmp-GFP pre-expression NO• consumption assay}

Translationally-fused Hmp-GFP was placed under the control of an IPTG-inducible $\mathrm{P}_{\mathrm{T} 5}$ promoter on a plasmid (pJR03), and transformed into $\Delta h m p$ and $\Delta h m p \Delta c l p P$ strains. The strains were grown identically as described for the $\mathrm{NO} \bullet$ consumption assay (section 4.6), except 15 min prior to the flask cultures reaching a $0.2 \mathrm{OD}_{600}, 50 \mu \mathrm{M}$ IPTG was delivered to induce expression of Hmp-GFP. After 15 min of induction, the cells were centrifuged and washed in fresh, prewarmed MOPS + glucose media containing $50 \mu \mathrm{g} / \mathrm{ml} \mathrm{CAM} \mathrm{to} \mathrm{prevent} \mathrm{further} \mathrm{protein} \mathrm{synthesis.}$ Meanwhile, the bioreactor was loaded with $10 \mathrm{ml}$ of fresh MOPS + glucose media (with 50 $\mu \mathrm{g} / \mathrm{ml} \mathrm{CAM}$ ), and dosed with $250 \mu \mathrm{M}$ DPTA NONOate, allowing the NO• to achieve a relatively stable concentration ( $10 \mathrm{~min}$ after dose) by the time the washing steps were complete. A portion of the CAM-treated cells were delivered to the $\mathrm{NO} \bullet$-treated media to a final $\mathrm{OD}_{600}$ of 0.05 , while another portion was diluted into a microplate $\left(\mathrm{OD}_{600}=0.2\right.$ to enhance signal intensity) to quantify GFP fluorescence. As controls, the assay was performed identically without induction (IPTG excluded), or using the empty plasmid (hmp-gfp coding sequence excluded) (Fig. S2).

\subsection{Hmp-GFP induction-tuning assay}

The IPTG-inducible, fused Hmp-GFP expression system described for the Hmp-GFP preexpression $\mathrm{NO} \bullet$ consumption assay (section 4.7) was transferred to a lower-copy plasmid backbone (pUA66 [57]) to facilitate tighter, more finely tuned expression of the protein (yielding the pJR05). The pJR05 plasmid was transformed into $\Delta h m p$ and $\Delta h m p \Delta c l p P$ to eliminate native hmp expression. The strains were grown and treated identically as described for the NO• consumption assay (section 4.6), except immediately after DPTA NONOate delivery, the cultures were induced with $10 \mu \mathrm{M}\left(\operatorname{clp} P^{+}\right)$or $1 \mathrm{mM}\left(\operatorname{clp} P^{-}\right)$IPTG. The NO• concentration was 
monitored continuously for $30 \mathrm{~min}$, while fluorescence was measured every $10 \mathrm{~min}$, and samples removed for RNA purification and quantification (qPCR) at 0,10 , and $30 \mathrm{~min}$. The $30 \mathrm{~min}$ fluorescence and mRNA concentration were not quantified for the $c l p P^{+}$culture, as the NO• was cleared by $20 \mathrm{~min}$, and growth had resumed by $30 \mathrm{~min}$.

\section{9 mRNA degradation rate assay}

A GFP expression plasmid was constructed by cloning $g f p$ into the pQE-80L vector (yielding pJR06), allowing IPTG-inducible expression from the $\mathrm{P}_{\mathrm{T} 5}$ promoter, and this was transformed into $\Delta h m p$ and $\Delta h m p \Delta c l p P$ strains. The strains were grown identically as described for the NO• consumption assay (section 4.6), but induced with $1 \mathrm{mM}$ IPTG for 15 minutes prior to centrifugation and washing. Cells were resuspended in fresh, pre-warmed $\left(37^{\circ} \mathrm{C}\right) \mathrm{MOPS}$ minimal media with $10 \mathrm{mM}$ glucose, and delivered to the bioreactor (to a final $\mathrm{OD}_{600}$ of 0.05 ) containing $10 \mathrm{ml}$ of fresh MOPS glucose media with $500 \mu \mathrm{g} / \mathrm{ml}$ rifampicin (RIF) to halt transcription. The bioreactor culture was then immediately dosed with $250 \mu \mathrm{M}$ DPTA NONOate, and GFP fluorescence was monitored every $5 \mathrm{~min}$ for the first $15 \mathrm{~min}$, followed by every $15 \mathrm{~min}$ for the next 45 min. Fluorescence values of identically-treated, but uninduced (no IPTG) cells were also obtained to be subtracted as background. In addition, a $500 \mu \mathrm{L}$ sample of culture was removed immediately prior to treatment at time zero for $g f p$ mRNA quantification using qPCR. Finally, to confirm that transcription was halted by RIF, the experiment was repeated identically except $1 \mathrm{mM}$ IPTG was added at the time of RIF treatment and DPTA NONOate dose, rather than 15 minutes prior, and fluorescence was monitored. A negligible increase in fluorescence over the course of the assay confirmed that transcription was sufficiently inhibited (Fig. S5). 


\subsection{0 $\mathrm{P}_{h m p}-\mathrm{g} f p$ reporter assay}

An hmp transcriptional reporter was constructed by placing $g f p$ under control of the $h m p$ promoter ( $\mathrm{P}_{h m p}, 250 \mathrm{bp}$ of the 5' UTR directly upstream of the hmp start codon) on the pUA66 plasmid [57] (yielding pJR04), and transformed into $\Delta h m p$ and $\Delta h m p \Delta c l p P$ strains. The cells were grown and treated with $250 \mu \mathrm{M}$ DPTA NONOate, identically as described for the NO• consumption assay (section 4.6). GFP fluorescence was measured every $0.5 \mathrm{~h}$. At $0 \mathrm{~h}$ (before dose), $0.5 \mathrm{~h}, 1 \mathrm{~h}$, and $2 \mathrm{~h}, 500 \mu \mathrm{L}$ of culture was removed for subsequent RNA purification and qPCR analysis.

\subsection{Real-time quantitative PCR (qPCR)}

\subsubsection{RNA purification}

To harvest RNA, $500 \mu \mathrm{L}$ of culture was removed and immediately mixed with $1 \mathrm{ml}$ of RNAprotect Bacteria Reagent (Qiagen) to stabilize the RNA. Purification was performed using the RNeasy Mini Kit (Qiagen), according to the manufacturer's protocol. Briefly, after 5 min incubation with the RNAprotect reagent, the sample was centrifuged for $10 \mathrm{~min}$ at $5000 \times g$, and the supernatant carefully discarded. Cell pellets were stored at $-80{ }^{\circ} \mathrm{C}$ until all replicates and conditions for that particular experiment were completed, allowing simultaneous purification of all samples. To account for variations in RNA loss among samples during the RNA purification process, $250 \mathrm{ng}$ of an external mRNA standard (preparation described below) was spiked into each of the thawed pellets immediately prior to purification, as described previously [58]. To eliminate potential DNA contamination, an on-column DNase digestion was performed using RNase-free DNase I (Qiagen). Sample purity and concentration were analyzed with a NanoDrop 1000 (Thermo Scientific), and stored at $-80^{\circ} \mathrm{C}$. 


\subsubsection{External mRNA standard synthesis}

The mRNA standard was a synthetically transcribed gene $(p h z M)$ from $P$. aeruginosa, selected based on its absence from the $E$. coli chromosome. The $p h z M$ gene was cloned from purified $P$. aeruginosa PAO1 genomic DNA, and inserted into the pET-11a plasmid, such that its expression was under the control of the T7 phage promoter [58]. The pET-11a-phzM plasmid was linearized by a $1 \mathrm{~h}$ restriction enzyme (RE) digest with EcoRI-HF ${ }^{\circledR}$ (New England Biolabs; NEB), and PCR-purified (Qiagen). The $p h z M$ mRNA was synthesized in vitro with the T7 High Yield RNA Synthesis Kit (NEB), using the linearized pET-11a-phzM plasmid as template, and the product mRNA was purified with the RNeasy Mini Kit (Qiagen), including an on-column DNase I digestion. Sample purity and concentration was measured on a NanoDrop 1000, and was stored at $-80^{\circ} \mathrm{C}$.

\subsection{3 cDNA synthesis}

Purified mRNA ( 100 ng per sample) was converted to cDNA using TaqMan ${ }^{\circledR}$ Reverse Transcription Reagents (Life Technologies) according to the manufacturer's protocol, with a 10 $\mu \mathrm{L}$ reaction volume, using random hexamers. As a control, a second set of reverse transcription (RT) reactions were run, excluding the reverse transcriptase enzyme, to check for DNA contamination of the sample.

\subsubsection{Real-time PCR}

Real-time PCR was performed using the SYBR ${ }^{\circledR}$ Green PCR Master Mix (Applied Biosystems) in an optical 96-well PCR plate, with $20 \mu 1$ reaction volumes consisting of $1 \mu \mathrm{l}$ cDNA from RT reaction, $250 \mathrm{nM}$ forward and reverse primers (Table S4), and $10 \mu 1$ of SYBR ${ }^{\circledR}$ Green PCR Master Mix. The PCR was run for 40 cycles using an ABI 7900 HT real-time thermocycler, and the $\mathrm{C}_{\mathrm{t}}$ (threshold cycle) values were obtained from the amplification curves. 
Each sample was run in separate reactions for $p h z M$ primers and target gene primers (and in biological triplicate or more), to allow quantification of $p h z M$ and target mRNA in each sample. To allow for absolute quantification of mRNA transcripts in each sample, standards were included on the PCR plate for each primer set, consisting of serial dilutions of the purified pET11a-phzM plasmid and the plasmid carrying the target gene, allowing generation of concentration vs. $\mathrm{C}_{\mathrm{t}}$ calibration curves. Target gene concentrations were normalized by $p h z M$ concentrations in each sample to allow comparison among the different samples.

\subsection{RNA sequencing (RNA-Seq)}

WT or $\Delta c l p P$ E. coli were grown and treated (or left untreated) with $250 \mu \mathrm{M}$ DPTA NONOate identically as described for the NO• consumption assay (section 4.6). After $10 \mathrm{~min}$ of treatment, $3 \mathrm{ml}$ of culture was removed and immediately mixed with $6 \mathrm{ml}$ of RNAprotect Bacteria Reagent (Qiagen). Total RNA was purified using the same procedure as described above for the qPCR analysis, except no $p h z M$ mRNA was delivered to the pellet prior to RNA purification. Each of the four conditions (WT and $\Delta c l p P$, untreated and treated) were performed in biological triplicate, yielding a total of 12 samples. Library preparation, sequencing, and data analysis are described in Text S1. The raw and processed RNA-Seq data from this publication have been submitted to the GEO database (http://www.ncbi.nlm.nih.gov/geo/) with accession number GSE60522. 


\subsection{Computational methods}

\subsubsection{Kinetic model description}

All model simulations, calculations, and parametric analyses were performed using MATLAB (R2012a, The MathWorks Inc.). The kinetic model of the E. coli NO• biochemical network used here was developed and described in previous studies [29,30]. Briefly, the model is comprised of a system of differential mass balances describing the change in species concentrations $(\boldsymbol{X})$ subject to a network of chemical reactions (organized into a stoichiometry matrix, $\boldsymbol{S})$, whose rates $(\boldsymbol{r})$ are a function of kinetic parameters $(p)$ and concentrations $(x)$ of individual species.

$$
\frac{d \boldsymbol{X}}{d t}=\boldsymbol{S} \cdot \boldsymbol{r}(x, p)
$$

The model was partitioned into a media and cellular compartment, to facilitate comparisons and parameterization with experimental measurements. The system of differential equations was integrated numerically using the MATLAB ode15s function, yielding species concentrations over the specified time interval.

To allow a more mechanistic interrogation of the $\triangle c l p P$ phenotype, protein synthesis, which was originally described by a single generation reaction, was partitioned into separate transcription and translation components. Production of mRNA transcripts was assumed to follow Hill-type kinetics, accounting for transcriptional regulation by fractional occupation of the relevant species [59], and degradation was assumed to follow first order decay kinetics [60,61]. The rates of protein translation were expressed as function of [mRNA] assuming elementary first-order kinetics, consistent with previous deterministic modeling studies [62-66]. The reaction 
equations, rate expressions, and parameter values for transcription and translation of Hmp, NorV, and NrfA are presented in Table S5, and the optimization of the new model components is described in Text S1 and Table S6.

\subsubsection{Model discrimination}

The ensemble of models was used to identify experimental conditions in silico that resulted in a large variation in predictions among the different $c l p P^{-}$models. We note that error associated with parameters can introduce uncertainty into model simulations, and could therefore

quantitatively change simulations where a difference between $c l p P^{+}$and $c l p P^{-}$has been observed (enhance or reduce differences). Such uncertainty would need to be accounted for if elimination of models was performed based on absolute quantitative values. However, in recognition of this uncertainty and its potential effects, the model discrimination process was confined to qualitative binary decisions based on statistical analysis of the experimental data (e.g., difference/no difference). In this manner, conclusions drawn regarding the validity of a specific mechanism were independent of variation that would propagate from uncertainty in parameter values.

\section{Acknowledgements}

We thank professors A. James Link, Stanislav Y. Shvartsman, Ned S. Wingreen, Thomas J. Silhavy, and James B. Kaper for their assistance and helpful discussions. EHEC TUV93-0 was kindly provided by Prof. John M. Leong, and we acknowledge the National BioResource Project (NIG, Japan) for distribution of the Keio collection. We also thank Dr. Wei Wang, Jennifer Miller, Jessica Wiggins, and Lance Parsons for their help with RNA-Seq. This work was supported by the National Science Foundation (CBET-1453325 and DGE-1148900), and Princeton University (Forese Family Fund for Innovation, start-up funds). 


\section{References}

1. Toledo JC, Augusto O (2012) Connecting the Chemical and Biological Properties of Nitric Oxide. Chem Res Toxicol 25: 975-989.

2. Nathan C (1992) Nitric Oxide as a Secretory Product of Mammalian Cells. FASEB J 6: 30513064.

3. Bowman LA, McLean S, Poole RK, Fukuto JM (2011) The diversity of microbial responses to nitric oxide and agents of nitrosative stress close cousins but not identical twins. Adv Microb Physiol 59: 135-219.

4. Fang FC (2004) Antimicrobial reactive oxygen and nitrogen species: Concepts and controversies. Nat Rev Microbiol 2: 820-832.

5. Yu H, Sato EF, Nagata K, Nishikawa M, Kashiba M, et al. (1997) Oxygen-dependent regulation of the respiration and growth of Escherichia coli by nitric oxide. FEBS Lett 409: 161-165.

6. Hyduke DR, Jarboe LR, Tran LM, Chou KJ, Liao JC (2007) Integrated network analysis identifies nitric oxide response networks and dihydroxyacid dehydratase as a crucial target in Escherichia coli. Proc Natl Acad Sci U S A 104: 8484-8489.

7. Richardson AR, Payne EC, Younger N, Karlinsey JE, Thomas VC, et al. (2011) Multiple targets of nitric oxide in the tricarboxylic acid cycle of Salmonella enterica serovar typhimurium. Cell Host Microbe 10: 33-43.

8. Kakishima K, Shiratsuchi A, Taoka A, Nakanishi Y, Fukumori Y (2007) Participation of nitric oxide reductase in survival of Pseudomonas aeruginosa in LPS-activated macrophages. Biochem Biophys Res Commun 355: 587-591.

9. Stern AM, Hay AJ, Liu Z, Desland FA, Zhang J, et al. (2012) The NorR Regulon Is Critical for Vibrio cholerae Resistance to Nitric Oxide and Sustained Colonization of the Intestines. MBio 3.

10. Darwin KH, Ehrt S, Gutierrez-Ramos JC, Weich N, Nathan CF (2003) The proteasome of Mycobacterium tuberculosis is required for resistance to nitric oxide. Science 302: 19631966.

11. Stevanin TM, Poole RK, Demoncheaux EAG, Read RC (2002) Flavohemoglobin Hmp protects Salmonella enterica serovar typhimurium from nitric oxide-related killing by human macrophages. Infect Immun 70: 4399-4405.

12. Svensson L, Poljakovic M, Save S, Gilberthorpec N, Schon T, et al. (2010) Role of flavohemoglobin in combating nitrosative stress in uropathogenic Escherichia coli Implications for urinary tract infection. Microb Pathog 49: 59-66.

13. Shimizu T, Tsutsuki H, Matsumoto A, Nakaya H, Noda M (2012) The nitric oxide reductase of enterohaemorrhagic Escherichia coli plays an important role for the survival within macrophages. Mol Microbiol 85: 492-512.

14. Robinson JL, Adolfsen KJ, Brynildsen MP (2014) Deciphering nitric oxide stress in bacteria with quantitative modeling. Curr Opin Microbiol 19C: 16-24.

15. Kulasekara BR, Jacobs M, Zhou Y, Wu ZN, Sims E, et al. (2009) Analysis of the Genome of the Escherichia coli O157:H7 2006 Spinach-Associated Outbreak Isolate Indicates Candidate Genes That May Enhance Virulence. Infect Immun 77: 3713-3721. 
16. MacMicking JD, North RJ, LaCourse R, Mudgett JS, Shah SK, et al. (1997) Identification of nitric oxide synthase as a protective locus against tuberculosis. Proc Natl Acad Sci U S A 94: 5243-5248.

17. MacMicking JD, Nathan C, Hom G, Chartrain N, Fletcher DS, et al. (1995) Altered Responses to Bacterial-Infection and Endotoxic-Shock in Mice Lacking Inducible NitricOxide Synthase. Cell 81: 641-650.

18. Chan J, Tanaka K, Carroll D, Flynn J, Bloom BR (1995) Effects of Nitric Oxide Synthase Inhibitors on Murine Infection with Mycobacterium tuberculosis. Infect Immun 63: 736740.

19. Bang IS, Liu LM, Vazquez-Torres A, Crouch ML, Stamler JS, et al. (2006) Maintenance of nitric oxide and redox homeostasis by the Salmonella flavohemoglobin Hmp. J Biol Chem 281: 28039-28047.

20. Stevanin TM, Ioannidis N, Mills CE, Kim SO, Hughes MN, et al. (2000) Flavohemoglobin Hmp affords inducible protection for Escherichia coli respiration, catalyzed by cytochromes $b o^{\prime}$ or $b d$, from nitric oxide. J Biol Chem 275: 35868-35875.

21. Helmick RA, Fletcher AE, Gardner AM, Gessner CR, Hvitved AN, et al. (2005) Imidazole antibiotics inhibit the nitric oxide dioxygenase function of microbial flavohemoglobin. Antimicrob Agents Chemother 49: 1837-1843.

22. Gur E, Ottofueling R, Dougan DA (2013) Machines of Destruction - AAA+ Proteases and the Adaptors That Control Them. In: Dougan DA, editor. Regulated Proteolysis in Microorganisms. New York: Springer. pp. 3-33.

23. Takaya A, Suzuki M, Matsui H, Tomoyasu T, Sashinami H, et al. (2003) Lon, a stressinduced ATP-Dependent protease, is critically important for systemic Salmonella enterica serovar typhimurium infection of mice. Infect Immun 71: 690-696.

24. Park CY, Kim EH, Choi SY, Tran TDH, Kim IH, et al. (2010) Virulence attenuation of Streptococcus pneumoniae clpP mutant by sensitivity to oxidative stress in macrophages via an NO-mediated pathway. J Microbiol 48: 229-235.

25. Madan-Lala R, Peixoto KV, Re F, Rengarajan J (2011) Mycobacterium tuberculosis Hip1 dampens macrophage proinflammatory responses by limiting toll-like receptor 2 activation. Infect Immun 79: 4828-4838.

26. Naffin-Olivos JL, Georgieva M, Goldfarb N, Madan-Lala R, Dong L, et al. (2014) Mycobacterium tuberculosis Hip1 Modulates Macrophage Responses through Proteolysis of GroEL2. PLoS Pathog 10.

27. Gaillot O, Pellegrini E, Bregenholt S, Nair S, Berche P (2000) The ClpP serine protease is essential for the intracellular parasitism and virulence of Listeria monocytogenes. Mol Microbiol 35: 1286-1294.

28. Jayasekera MM, Foltin SK, Olson ER, Holler TP (2000) Escherichia coli requires the protease activity of FtsH for growth. Arch Biochem Biophys 380: 103-107.

29. Robinson JL, Brynildsen MP (2013) A kinetic platform to determine the fate of nitric oxide in Escherichia coli. PLoS Comput Biol 9: e1003049.

30. Robinson JL, Miller RV, Brynildsen MP (2014) Model-Driven Identification of Dosing Regimens that Maximize the Antimicrobial Activity of Nitric Oxide. Metab Eng Commun 1: 12-18.

31. Pullan ST, Gidley MD, Jones RA, Barrett J, Stevanin TA, et al. (2007) Nitric oxide in chemostat-cultured Escherichia coli is sensed by Fnr and other global regulators: 
Unaltered methionine biosynthesis indicates lack of S nitrosation. J Bacteriol 189: 18451855.

32. Pesavento C, Becker G, Sommerfeldt N, Possling A, Tschowri N, et al. (2008) Inverse regulatory coordination of motility and curli-mediated adhesion in Escherichia coli. Genes Dev 22: 2434-2446.

33. Keseler IM, Mackie A, Peralta-Gil M, Santos-Zavaleta A, Gama-Castro S, et al. (2013) EcoCyc: fusing model organism databases with systems biology. Nucleic Acids Research 41: D605-612.

34. Centers for Disease Control and Prevention (CDC) (2013) Antibiotic Resistance Threats in the United States, 2013, CS239559-B. Available: http://www.cdc.gov/drugresistance/threat-report-2013. Accessed 27 August 2014.

35. Bush K, Courvalin P, Dantas G, Davies J, Eisenstein B, et al. (2011) Tackling antibiotic resistance. Nat Rev Microbiol 9: 894-896.

36. Talbot GH, Bradley J, Edwards JE, Jr., Gilbert D, Scheld M, et al. (2006) Bad bugs need drugs: an update on the development pipeline from the Antimicrobial Availability Task Force of the Infectious Diseases Society of America. Clin Infect Dis 42: 657-668.

37. Fernebro J (2011) Fighting bacterial infections-future treatment options. Drug Resist Updat 14: $125-139$.

38. Cegelski L, Marshall GR, Eldridge GR, Hultgren SJ (2008) The biology and future prospects of antivirulence therapies. Nat Rev Microbiol 6: 17-27.

39. Rasko DA, Sperandio V (2010) Anti-virulence strategies to combat bacteria-mediated disease. Nat Rev Drug Discov 9: 117-128.

40. Dethlefsen L, Relman DA (2011) Incomplete recovery and individualized responses of the human distal gut microbiota to repeated antibiotic perturbation. Proc Natl Acad Sci U S A 108: 4554-4561.

41. Michel A, Agerer F, Hauck CR, Herrmann M, Ullrich J, et al. (2006) Global regulatory impact of ClpP protease of Staphylococcus aureus on regulons involved in virulence, oxidative stress response, autolysis, and DNA repair. J Bacteriol 188: 5783-5796.

42. Iyoda S, Watanabe H (2005) ClpXP protease controls expression of the type III protein secretion system through regulation of RpoS and GrlR levels in enterohemorrhagic Escherichia coli. J Bacteriol 187: 4086-4094.

43. Compton CL, Schmitz KR, Sauer RT, Sello JK (2013) Antibacterial activity of and resistance to small molecule inhibitors of the ClpP peptidase. ACS Chem Biol 8: 2669-2677.

44. McGillivray SM, Tran DN, Ramadoss NS, Alumasa JN, Okumura CY, et al. (2012) Pharmacological inhibition of the ClpXP protease increases bacterial susceptibility to host cathelicidin antimicrobial peptides and cell envelope-active antibiotics. Antimicrob Agents Chemother 56: 1854-1861.

45. Wang L, Hatzimanikatis V (2006) Metabolic engineering under uncertainty. I: framework development. Metab Eng 8: 133-141.

46. Wang L, Hatzimanikatis V (2006) Metabolic engineering under uncertainty - II: Analysis of yeast metabolism. Metab Eng 8: 142-159.

47. Kuepfer L, Peter M, Sauer U, Stelling J (2007) Ensemble modeling for analysis of cell signaling dynamics. Nat Biotechnol 25: 1001-1006.

48. Tran LM, Rizk ML, Liao JC (2008) Ensemble Modeling of Metabolic Networks. Biophys J 95: 5606-5617. 
49. Rizk ML, Liao JC (2009) Ensemble Modeling for Aromatic Production in Escherichia coli. Plos One 4.

50. Mišković L, Hatzimanikatis V (2011) Modeling of uncertainties in biochemical reactions. Biotechnology and Bioengineering 108: 413-423.

51. Jia G, Stephanopoulos G, Gunawan R (2012) Ensemble kinetic modeling of metabolic networks from dynamic metabolic profiles. Metabolites 2: 891-912.

52. Brynildsen MP, Winkler JA, Spina CS, MacDonald IC, Collins JJ (2013) Potentiating antibacterial activity by predictably enhancing endogenous microbial ROS production. Nat Biotechnol 31: 160-165.

53. Zomorrodi AR, Lafontaine Rivera JG, Liao JC, Maranas CD (2013) Optimization-driven identification of genetic perturbations accelerates the convergence of model parameters in ensemble modeling of metabolic networks. Biotechnol J 8: 1090-1104.

54. Khodayari A, Zomorrodi AR, Liao JC, Maranas CD (2014) A kinetic model of Escherichia coli core metabolism satisfying multiple sets of mutant flux data. Metab Eng 25: 50-62.

55. Lee Y, Lafontaine Rivera JG, Liao JC (2014) Ensemble Modeling for Robustness Analysis in engineering non-native metabolic pathways. Metab Eng 25: 63-71.

56. Adolfsen KJ, Brynildsen MP (2015) Futile cycling increases sensitivity toward oxidative stress in Escherichia coli. Metab Eng 29: 26-35.

57. Zaslaver A, Bren A, Ronen M, Itzkovitz S, Kikoin I, et al. (2006) A comprehensive library of fluorescent transcriptional reporters for Escherichia coli. Nat Methods 3: 623-628.

58. Amato SM, Brynildsen MP (2014) Nutrient Transitions Are a Source of Persisters in Escherichia coli Biofilms. PLoS One 9.

59. Sauro HM (2012) Enzyme Kinetics for Systems Biology. Seattle, WA: Ambrosius Publishing.

60. Laalami S, Zig L, Putzer H (2014) Initiation of mRNA decay in bacteria. Cell Mol Life Sci 71: 1799-1828.

61. Selinger DW, Saxena RM, Cheung KJ, Church GM, Rosenow C (2003) Global RNA halflife analysis in Escherichia coli reveals positional patterns of transcript degradation. Genome Res 13: 216-223.

62. Usuda Y, Nishio Y, Iwatani S, Van Dien SJ, Imaizumi A, et al. (2010) Dynamic modeling of Escherichia coli metabolic and regulatory systems for amino-acid production. J Biotechnol 147: 17-30.

63. Lee MV, Topper SE, Hubler SL, Hose J, Wenger CD, et al. (2011) A dynamic model of proteome changes reveals new roles for transcript alteration in yeast. Mol Syst Biol 7.

64. Peercy BE, Cox SJ, Shalel-Levanon S, San KY, Bennett G (2006) A kinetic model of oxygen regulation of cytochrome production in Escherichia coli. J Theor Biol 242: 547-563.

65. Belle A, Tanay A, Bitincka L, Shamir R, O'Shea EK (2006) Quantification of protein halflives in the budding yeast proteome. Proc Natl Acad Sci U S A 103: 13004-13009.

66. Nishio Y, Usuda Y, Matsui K, Kurata H (2008) Computer-aided rational design of the phosphotransferase system for enhanced glucose uptake in Escherichia coli. Mol Syst Biol 4. 